\title{
Mīmāmesā deontic reasoning using specificity: a proof theoretic approach
}

\author{
Björn Lellmann ${ }^{1}$ (D) $\cdot$ Francesca Gulisano $^{2} \cdot$ Agata Ciabattoni $^{1}$
}

Accepted: 13 October 2020 / Published online: 9 November 2020

(c) The Author(s) 2020

\begin{abstract}
Over the course of more than two millennia the philosophical school of Mīmāmsā has thoroughly analyzed normative statements. In this paper we approach a formalization of the deontic system which is applied but never explicitly discussed in Mīmāmsā to resolve conflicts between deontic statements by giving preference to the more specific ones. We first extend with prohibitions and recommendations the non-normal deontic logic extracted in Ciabattoni et al. (in: TABLEAUX 2015, volume 9323 of LNCS, Springer, 2015) from Mīmāmsā texts, obtaining a multimodal dyadic version of the deontic logic MD. Sequent calculus is then used to close a set of prima-facie injunctions under a restricted form of monotonicity, using specificity to avoid conflicts. We establish decidability and complexity results, and investigate the potential use of the resulting system for Mīmāmsāa philosophy and, more generally, for the formal interpretation of normative statements.
\end{abstract}

Keywords Deontic logic $\cdot$ Non-monotonic inference $\cdot$ Specificity $\cdot$ Mīmāmsāā Sequent systems $\cdot$ Legal representation

We thank E. Freschi for her invaluable help with the Indological aspects of this work. Funded by the Vienna Science and Technology Fund (WWTF) under project MA16-28 and by BRISE-Vienna (UIA04-081), a European Union Urban Innovative Actions project.

\footnotetext{
$\varangle$ Björn Lellmann

lellmann@logic.at

Francesca Gulisano

francesca.gulisano@sns.it

Agata Ciabattoni agata@logic.at

1 TU Wien, Vienna, Austria

2 Scuola Normale Superiore Pisa, Pisa, Italy
} 


\section{Introduction}

The Mīmāmsā is a philosophical school which originated in ancient India in the last centuries BCE and whose main focus was the exegesis of the prescriptive portions of the Vedas - the Sacred Texts of (what is now called) Hinduism. Together with Nyāya and Buddhist epistemology, Mīmāmsā is one of the fundamental schools of Indian philosophy, and the only one centered on deontic concepts. In order to read the Vedas not as a religious text, but as a set of precepts, and to explain "what has to be done" in presence of seemingly ${ }^{1}$ conflicting obligations, Mīmāṃsā authors have thoroughly discussed and analyzed normative statements. They have proposed a rich body of deontic, hermeneutical and linguistic principles of interpretation, called nyāyas, which are so modern, rational, scientific, and systematic (Bathia 2010) that they are still applied in Indian jurisprudence, e.g. Katju (2006). Although not well known to the logic community, the resulting theories are rightly considered early deontic logic (Huisjes 1981).

Among the deontic nyāyas, some can be transformed into properties (Hilbert axioms) for the operators corresponding to the deontic concepts in Mīmāmsāa this method led to the introduction of the non-normal dyadic deontic logic bMDL (basic Mìmāmsā deontic logic), which was used in Ciabattoni et al. (2015) to formally analyze a famous controversial passage in the Vedas. However, in the construction of bMDL only nyāyas concerning the obligation operator were considered. Here we extend bMDL with new operators for prohibitions and recommendations (or weak obligations); we call the resulting logic MD+. We extracted the properties for these operators from additional nyāyas that were translated from Sanskrit and interpreted only recently. Similar to the situation in Talmudic logic as investigated in Abraham et al. (2011), the Mīmāṃsā deontic operators are not interdefinable. Intuitively, the most evident difference between them is in the achievable results: obeying Vedic obligations yields good karma which leads to eternal happiness; in contrast, following Vedic recommendations yields only specific immediate results; finally, following Vedic prohibitions only prevents the accumulation of negative karma, see, e.g., Freschi (2012, 2017). However, the main difference between the two deontic concepts of prescription (vidhi in Sanskrit) and prohibition (nisedha) is not properly dependent on the results of complying with commands or disregarding them. The conveyed idea (buddhi) at the base of the concept of obligation is "activation" or "being impelled to act", while, in case of prohibition, it is "inhibition" or "being prevented from taking an action", hence prescription and prohibition represent two genuinely different notions of duty, one irreducible to the other

Although specifically targeted at formalizing Mīmāmsā reasoning, these operators can be applied in different contexts. For instance, in line with the argument for using deontic notions in the formalization of legal texts given in Jones and Sergot (1992), Royakkers (1998), obligations and prohibitions could be used for comparing moral and legal duties (see also Example 4 in Sect. 6), and the distinction between obligations and recommendations could be adapted for representing the difference between

\footnotetext{
${ }^{1}$ Since the Vedas are assumed not to be contradictory, Mīmāṃsā authors invested all their efforts in creating a consistent deontic system.
} 
prescriptions to fulfil duties within mandatory terms and within indicative terms, in some legal frameworks.

Not all the nyāyas can be converted into Hilbert axioms though. Some of these offer indeed more general interpretative principles to resolve apparent contradictions in the Vedas; prominent examples of such nyāyas are gunapradhāna (also known as sāmānya-viśeșa) and vikalpa, which are investigated in this paper. The vikalpa principle states that when there is a real conflict between obligations, any of the conflicting injunctions may be adopted as option: this principle is known in deontic logic as disjunctive response (Goble 2013) and is similar to the phenomenon of floating conclusions in nonmonotonic reasoning (Makinson and Schlechta 1991, see also Remark 7). Introduced by Śabara (3rd-5th c. CE), the gunapradhāna principle states that more specific rules override more generic ones; it is widely used, e.g., in Artificial Intelligence, where it is known as specificity principle, and in Law as the principle "Lex specialis derogat legi generali". These principles are also used to capture defeasible reasoning in the context of non-monotonic logics (see e.g. Delgrande and Schaub 1997; Nute 2003; Hage 2003; Straßer and Antonelli 2016).

Different methods and systems have been introduced in the literature to deal with deontic conflict resolutions using specificity. Although some are close to ours [e.g. Horty's syntactic approach in Horty (2012)], none of the various proposals can be used "out of the box" for representing Mīmāmsā reasoning. Indeed they are either based on logics different from MD+ (e.g. Straßer and Arieli 2019), or are implemented within general frameworks that do not allow us to distinguish between Vedic commands and human deductions [e.g. the argumentation-based approach in Prakken and Sartor (1999), and Deontic Default logic in Horty (1993, 2012), see Remark 3], use explicit priorities among rules_which are not present in Mīmāmsāa-[e.g., Defeasible Deontic logic in Governatori and Rotolo (2004) and Input/Output logic in Makinson and van der Torre (2000)], or a different way to apply specificity [e.g. Horty's approach (Horty 2012), see Example 2]. Due to its relevance to legal reasoning, a number of these approaches to conflict resolution have been applied in that area, and often implementations are available. Good recent overviews and comparisons are given, e.g., in Batsakis et al. (2018) and Calegari et al. (2019).

The aim of this article is to extend the basic deontic logic MD+ for obligations, prohibitions and recommendations with reasoning from deontic assumptions using specificity and vikalpa. We further explore the usefulness of the resulting system for the evaluation of different competing formalisations in Mīmāmsāa and beyond.

To this end we introduce a sequent-based approach to deal in MD+ with specificity and vikalpa as well as a system implementation. This work is a significantly extended version of the conference paper Ciabattoni et al. (2018), which only concerned obligations, and did not discuss potential applications.

Resolving conflicts using specificity, our calculus derives enforceable and applicable commands from the explicit prescriptions contained in the Vedas (śrauta in Sanskrit) and from a finite set of propositional facts.

The calculus presented here is also shown to satisfy vikalpa (disjunctive response). As, e.g., in Goble (2013), and van der Torre (1994), here we interpret the notion of a conditional obligation being more specific than another one as the conditions of the former implying those of the latter. Our calculus is built on the sequent calculus for 
the $\square$-free fragment of bMDL from Ciabattoni et al. (2015), which turns out to be the dyadic version of the non-normal deontic logic MD considered, e.g., in Chellas (1980) (cf. Proposition 1 and Freschi et al. 2019), extended with new rules for recommendations and prohibitions. Additional rules to derive all possible prescriptions are defined using limited (downwards) monotonicity on the conditions of the (non-nested) prescriptions in the Vedas (prima-facie deontic statements) "up to conflicting deontic statements" relative to the given set of facts. These rules offer the technical advantage that the consequences of a set of prima-facie deontic statements can be constructed iteratively instead of by a fixed-point construction, as, e.g., in Horty (2012). Importantly, since the prima-facie injunctions are assumed to constitute a closed set, the additional rules contain statements expressing the underivability of a formula; these statements do not compromise the decidability of the system and do not affect its complexity, which remains PSPACE, i.e., as for deciding theoremhood in intuitionistic or many standard modal logics. The central technical result ensuring these properties is the cut elimination theorem (Theorem 2). Similar underivability statements are present, e.g., in the sequent calculi for non-monotonic (non-modal) logics of Bonatti and Olivetti (2002), but in contrast to that work here we do not need to develop a full-fledged calculus for these statements. Other sequent-based calculi capturing non-monotonicity in the context of normative reasoning have been developed and applied in deontic logics (e.g. Governatori and Rotolo 2006) and in argument-based systems (e.g. Straßer and Arieli 2019).

The design of our system is motivated by the particular interpretation given by most of Mīmāmsā authors and made explicit by the later author Medhātithi (9-10th c. CE), that more specific śrauta precepts provide exceptions to more general ones and that the latter apply to all circumstances but those indicated in the exceptions (or implied by them). Apart from the nonmonotonic inferences from prima-facie to actual deontic statements, all derivations use the monotonic system MD+. Keeping the inferences of the logic at this level deductive (i.e., monotone) is inspired by the effort of Indian philosophers — in particular the Mīmāmssā author Kumārila— to keep their arguments not defeasible "as much as possible", see Taber (2004).

Applications of our system to Mīmāmsā philosophy, and, more generally, to the formal interpretation of normative statements, e.g., in legal representation, are also provided. Prima-facie (śrauta) injunctions and statements about factual conditions can indeed give rise to many interpretations, each of which corresponds to a group of prima-facie commands and global assumptions about facts. For instance, in Sanskrit the same word is used both for "obligations" and "recommendations", and the correct meaning has to be inferred by scholars of Indian philosophy. Our system can be used to derive a set of consequences for each of these groups. Using these consequences to compare the different interpretations, it is then possible to choose the most suitable one. In the case of Mīmāmsā philosophy, one criterium which was heavily used in this comparison is to minimize applications of the vikalpa principle between primafacie deontic statements; this is due to the fact that Mīmāmsāa authors considered applications of vikalpa to be "the last resort" and hence to be avoided as much as possible. In our system this criterium can be evaluated by checking how many of the prima-facie deontic statements are actually derivable. This check can be performed 
with the help of our Prolog implementation of the system, available at http://subsell. logic.at/bprover/deonticProver/version1.2/.

The rest of the paper is organized as follows: Sect. 2 recalls the base logic bMDL. The simplified set of sequent calculus rules, from Ciabattoni et al. (2018), to reason about obligations in presence of specificity is given in Sect. 3. The base logic is extended with prohibitions and recommendations in Sect. 4, and the complete set of rules for reasoning using specificity is introduced in Sect. 5. Consistency, decidability and complexity results for the system are presented in Sect. 5.1, while its potential use for Mīmāmsā philosophy and beyond is described in Sect. 6. The technical proof of cut elimination is contained in the "Appendix".

\section{The base logic: bMDL}

Basic Mīmāmsā Deontic Logic bMDL was introduced in Ciabattoni et al. (2015) as a first step towards mapping the structural elements of the Mīmāmsā deontic system onto a formal framework. The idea was to define a logical system following a bottom-up approach of extracting deontic principles from the Mīmāmsā texts. The logic resulting from the analysis of circa 50 such principles extends the alethic system S4 with the following axiom schemata for the deontic operator $\mathcal{O}(A / B)$, which intuitively reads as " $A$ is obligatory under the condition $B$ ":

1. $(\square(A \rightarrow B) \wedge \mathcal{O}(A / C)) \rightarrow \mathcal{O}(B / C)$.

2. $\square(B \rightarrow \neg A) \rightarrow \neg(\mathcal{O}(A / C) \wedge \mathcal{O}(B / C))$.

3. $(\square((B \rightarrow C) \wedge(C \rightarrow B)) \wedge \mathcal{O}(A / B)) \rightarrow \mathcal{O}(A / C)$.

Axioms (1)-(3) arise by rewriting some of the Mīmāmsā deontic interpretative principles (nyāyas) as logic formulae, while the choice of modal logic S4 over S5 was suggested by some statements found in the texts as well as technical convenience, in particular the existence of cut-free sequent calculi. See Ciabattoni et al. $(2017,2015)$ for more details.

Note that deontic statements in Mīmāmsā can also be analysed on a more detailed level in the context of specific sacrifices, taking into account the nature of the latter. Since here we are interested in the general properties of Mīmāmsā reasoning, we do not consider this distinction and refer the reader to Freschi et al. (2019) for details.

Remark 1 bMDL is weaker than most known deontic logics, e.g., the logics considered in von Wright (1964), von Wright (1965), Hansson (1969), van Fraassen (1972), Prakken and Sergot (1997), Goble (2019); in particular it has neither any deontic aggregation principles like $\mathcal{O}(A / C) \wedge \mathcal{O}(B / C) \rightarrow \mathcal{O}(A \wedge B / C)$ nor any form of factual or deontic detachment, i.e., $\mathcal{O}(A / B) \wedge B \rightarrow \mathcal{O}(A)$ and $\mathcal{O}(A / B) \wedge \mathcal{O}(B / C) \rightarrow$ $\mathcal{O}(A / C)$ respectively. In part this is due to our bottom-up methodology: so far indeed we have not found any mention of corresponding principles in the texts. However, the absence of (factual) detachment principles is also in line with the statement by one of the main authors of Mīmāmsā, Prabhākara, that "A prescription regards what has to be done. But it does not say that it has to be done" (Brhatī I, 7th c. CE). We read this as stating that a prescription states what is obligatory under certain conditions, but not that this is unconditionally obligatory if these conditions hold. 


$$
\begin{array}{ll}
\text { (M) } \mathcal{O}(A \wedge B / C) \rightarrow \mathcal{O}(A / C) & \frac{A \leftrightarrow C \quad B \leftrightarrow D}{\mathcal{O}(A / B) \rightarrow \mathcal{O}(C / D)} \mathrm{Cg} \\
\text { (D) } \neg(\mathcal{O}(A / B) \wedge \mathcal{O}(\neg A / B)) &
\end{array}
$$

Fig. 1 The modal part of a Hilbert-style system for dyadic MD

$$
\begin{gathered}
\frac{}{A \Rightarrow A} \text { init } \frac{\Gamma, B \Rightarrow \Delta \quad \Gamma \Rightarrow A, \Delta}{\perp,} \perp_{L} \quad \frac{\Gamma, A \Rightarrow B, \Delta}{\Gamma \Rightarrow A \rightarrow B, \Delta} \rightarrow_{R} \\
\frac{A \Rightarrow C \quad B \Rightarrow D \quad D \Rightarrow B}{\mathcal{O}(A / B) \Rightarrow \mathcal{O}(C / D)} \operatorname{Mon}_{\mathcal{O}} \frac{A, C \Rightarrow \quad B \Rightarrow D \quad D \Rightarrow B}{\mathcal{O}(A / B), \mathcal{O}(C / D) \Rightarrow} \mathrm{D}_{\mathcal{O}} \frac{A \Rightarrow}{\mathcal{O}(A / B) \Rightarrow} \mathrm{P}_{\mathcal{O}} \\
\frac{\Gamma, A, A \Rightarrow \Delta}{\Gamma, A \Rightarrow \Delta} \operatorname{Con}_{L} \frac{\Gamma \Rightarrow A, A, \Delta}{\Gamma \Rightarrow A, \Delta} \operatorname{Con}_{R} \frac{\Gamma \Rightarrow \Delta}{\Gamma, A \Rightarrow \Delta} \mathrm{W}_{L} \frac{\Gamma \Rightarrow \Delta}{\Gamma \Rightarrow A, \Delta} \mathrm{W}_{R}
\end{gathered}
$$

Fig. 2 The sequent calculus $G_{M D}$ for dyadic MD

$$
\begin{gathered}
\frac{\Gamma^{\square} \Rightarrow A}{\Gamma \Rightarrow \square A, \Delta} 4 \quad \frac{\Gamma, \square A, A \Rightarrow \Delta}{\Gamma, \square A \Rightarrow \Delta} \mathrm{T} \quad \frac{\Gamma^{\square}, A \Rightarrow C \quad \Gamma^{\square}, B \Rightarrow D \quad \Gamma^{\square}, D \Rightarrow B}{\Gamma, \mathcal{O}(A / B) \Rightarrow \mathcal{O}(C / D), \Delta} \text { Mon' }^{\prime} \\
\frac{\Gamma^{\square}, A \Rightarrow}{\Gamma, \mathcal{O}(A / B) \Rightarrow \Delta} \mathrm{D}_{1} \quad \frac{\Gamma^{\square}, A, C \Rightarrow \quad \Gamma^{\square}, B \Rightarrow D \quad \Gamma^{\square}, D \Rightarrow B}{\Gamma, \mathcal{O}(A / B), \mathcal{O}(C / D) \Rightarrow \Delta} \mathrm{D}_{2}
\end{gathered}
$$

Fig. 3 The modal part of the sequent calculus $\mathrm{G}_{\mathrm{bMDL}}$ for bMDL from Ciabattoni et al. (2015)

Here for simplicity we only consider the box-free fragment of bMDL. Formally, the set of formulae is given by the grammar $A::=p|\perp| A \rightarrow A \mid \mathcal{O}(A / A)$. We treat the remaining propositional connectives $\wedge, \vee, \neg$ as defined by $\{\perp, \rightarrow\}$ in the usual way, i.e., $\neg A: \equiv A \rightarrow \perp$ as well as $A \vee B: \equiv \neg A \rightarrow B$ and $A \wedge B: \equiv \neg(\neg A \vee \neg B)$. We will show in Proposition 1 below that the box-free fragment of bMDL coincides with the dyadic version of the logic MD (see Chellas 1980) axiomatized as in Fig. 1

In this paper we will consider an extension of a sequent calculus for this logic. Here, a sequent is a tuple of multisets ${ }^{2}$ of formulae, written as $\Gamma \Rightarrow \Delta$. The rules of the base sequent calculus $\mathrm{G}_{\mathrm{MD}}$ are given in Fig. 2, those of the calculus $\mathrm{G}_{\mathrm{bMDL}}$ for bMDL from Ciabattoni et al. (2015) in Fig. 3, where $\Gamma^{\square}$ denotes $\Gamma$ in which all formulae not of the form $\square A$ are deleted. As usual, a derivation is a finite labelled tree where every node is labelled with a sequent such that the labels of a node follow from the labels of its children using the rules of the calculus. In particular, the leaves are labelled with conclusions of the zero-premise rules init or $\perp_{L}$, see also Troelstra and Schwichtenberg (2000). For $G$ one of $G_{M D}, G_{b M D L}$ we write $\vdash_{G} \Gamma \Rightarrow \Delta$ if there is a derivation of $\Gamma \Rightarrow \Delta$ in $\mathrm{G}$. The following proposition gives a proof-theoretic proof of the equivalence of the box-free fragment of bMDL and MD. For the original proof using semantical methods, see Freschi et al. (2019).

Proposition 1 If $\Gamma \Rightarrow \Delta$ does not contain $\square$, then $\vdash_{G_{M D}} \Gamma \Rightarrow \Delta$ iff $\vdash_{G_{b M D L}} \Gamma \Rightarrow \Delta$. Hence the box-free fragment of bMDL is MD.

Proof One direction of the equivalence follows from changing the rules of $\mathrm{G}_{\mathrm{bMDL}}$ into the corresponding rules of $\mathrm{G}_{\mathrm{MD}}$ possibly followed by the weakening rules $\mathrm{W}_{L}, \mathrm{~W}_{R}$.

\footnotetext{
2 Note that since we have contraction on both sides of the sequent we could alternatively consider sequents as tuples of sets instead of multisets. To make the role of contraction explicit and to facilitate a less errorprone cut elimination proof, we chose to use multisets.
} 
The other direction follows since a derivation in $G_{M D}$ is a derivation in $G_{b M D L}$ with the addition of the structural rules of weakening and contraction $\operatorname{Con}_{L}, \operatorname{Con}_{R}$, which are admissible in $G_{b M D L}$ (Ciabattoni et al. 2015, Lemma 1). Completeness and soundness of $G_{M D}$ for MD follow from general methods (e.g. in Lellmann and Pattinson 2013) for constructing sequent calculi from axioms and proving cut elimination.

Remark 2 Following Ciabattoni et al. (2018), in this paper we employ a mechanism for handling propositional facts that differs from that in Ciabattoni et al. (2015): whereas there we encoded such assumptions as boxed formulae in the conclusion of a derivation, here we treat them as leaves in a derivation. E.g., for deriving that the conditional obligation to not perform violence implies the conditional obligation to not kill from the assumption that killing implies violence, using the mechanism from Ciabattoni et al. (2015) we would try to derive the sequent $\square$ (kill $\rightarrow$ violence), $\mathcal{O}(\neg$ violence $/ C) \Rightarrow$ $\mathcal{O}(\neg$ kill $/ C)$ using only inital sequents at the leaves of the derivation. Here, instead we will try to derive the sequent $\mathcal{O}(\neg$ violence $/ C) \Rightarrow \mathcal{O}(\neg$ kill $/ C)$, where the non-logical axiom or ground sequent kill $\Rightarrow$ violence may occur at a leaf of the derivation (see Definition 1 below for the formal details). This has the welcome consequence that we can avoid the alethic modality $\square$ including any question about its axiomatisation, in line with the view that Mīmāmsā authors did not distinguish between necessity and epistemic certainty.

\section{Reasoning with more specific obligations in Mīmāṃsā}

Here we continue the proof-theoretic approach initiated in Ciabattoni et al. (2015) to reproduce Mīmāmsā reasoning in a formal framework.

Before considering the full language, we first illustrate the main ideas behind the sequent calculus approach to deal with specificity/gunapradhāna in the simplified context of Ciabattoni et al. (2018), i.e. using the (dyadic version of the) logic MD with the obligation operator only.

Specificity is used in Mīmāṃsā to resolve apparent contradictions which may occur in the set of Vedic (śrauta) prescriptions or can be derived via the facts. For example, consider the śrauta injunctions: (a) You ought not to study the Vedas if you are a Śūdra (i.e., a member of the lower class), (b) Not studying the Vedas implies not performing the Agnihotra sacrifice, and (c) You ought to perform the Agnihotra if you are a chariot maker. The additional fact (d) A chariot maker is a Súdra, (apparently) leads to the conflicting obligations that you ought to study the Vedas if you are a chariot maker and that at the same time you ought not to do so, as extensively discussed by the Mīmāṃā author Jaimini (2nd c. BCE). The following example illustrates the way Mīmāmsāa authors reason to solve such kinds of conflicting obligations. Moreover it shows that inferences which aim to mimic Mīmāmsā reasoning do not satisfy some of the principles identified as key properties of non-monotonic logics in Gabbay (1985).

Remark 3 A central feature of our calculus is the distinction between prima facie and derived obligations, needed for differentiating Vedic commands from human deductions. This distinction is instead missing in Deontic Default logic (Horty 2012), that also does not satisfy the Vikalpa principle. 
From now on we will denote prima-facie obligations or deontic assumptions (śrauta obligations) with $\mathcal{O}_{\mathrm{pf}}(A / B)$ to distinguish them from derived obligations (written $\mathcal{O}(A / B))$. Formally, the language of deontic assumptions is obtained from the language of MD by replacing the operator $\mathcal{O}$ with its prima-facie variant $\mathcal{O}_{\text {pf }}$.

Example 1 We formalize the above statements concerning the Agnihotra sacrifice as follows : (a) $\mathcal{O}_{\mathrm{pf}}(\neg \mathrm{ved} / \mathrm{sdr})$, (b) agn $\rightarrow$ ved, (c) $\mathcal{O}_{\mathrm{pf}}(\mathrm{agn} / \mathrm{chmk})$, and (d) chmk $\rightarrow$ sdr, where ved denotes the act of studying the Vedas, agn the performance of the Agnihotra sacrifice, sdr the fact of being a Śūdra, and chmk being a chariot maker. Using the monotonicity of the deontic operator in its first argument, from (b) and (c) we derive the obligation (e) $\mathcal{O}$ (ved/chmk) ("You ought to study the Vedas if you are a chariot maker"). On the other hand, if it were possible to use indiscriminately monotonicity in the second argument of the deontic operator, from (a) and (d) we would derive (f) $\mathcal{O}$ ( $\neg$ ved/chmk) ("You ought not to study the Vedas if you are a chariot maker"), obtaining a conflict between (e) and (f).

By applying the specificity principle, we "limit" the monotonicity in the second argument of the operator; hence, following the above example, the derivation of (f) is blocked by the presence of the prima-facie obligation (c).

The derivations from prima-facie injunctions are non-monotonic, as adding more premisses can change the derived result. However, they do not satisfy for example cautious monotonicity (if $\Gamma \vdash \varphi$ and $\Gamma \vdash \psi$, then $\Gamma, \varphi \vdash \psi$ ), one of the crucial properties of non-monotonic logics. Indeed given the prima-facie injunctions $\mathcal{O}_{\text {pf }}(\mathrm{ved} / \top)$ and $\mathcal{O}_{\text {pf }}(\neg \mathrm{ved} / \mathrm{sdr})$, both $\mathcal{O}$ (ved/tch) ("You ought to study the Vedas if you are a teacher") and $\mathcal{O}(\neg \mathrm{ved} / \mathrm{tch} \wedge \mathrm{sdr})$ are derivable, but, if one of these conclusions is considered as a premiss, the result changes, i.e. $\left\{\mathcal{O}_{\text {pf }}(\right.$ ved $/ \top), \mathcal{O}_{\text {pf }}(\neg$ ved/sdr $), \mathcal{O}_{\text {pf }}($ ved/tch $\left.)\right\} \nvdash \mathcal{O}(\neg$ ved/tch $\wedge$ sdr $)$.

We extend below the sequent calculus $G_{M D}$ for the logic MD with special rules $\mathcal{O}_{L}^{\mathcal{O}_{\mathrm{pf}}(C / D)}, \mathcal{O}_{R}^{\mathcal{O}_{\mathrm{pf}}(C / D)}$ to derive conditional obligations of the form $\mathcal{O}(A / B)$ from prima-facie obligations (i.e. śrauta prescriptions) written as $\mathcal{O}_{\mathrm{pf}}(C / D)$, adopting limited forms of monotonicity. (Sect. 3.1). The extension to the full language will be considered in Sect. 4.

\subsection{Sequent calculus for specificity/gunapradhāna}

In order to extend the sequent calculus for MD to capture the specificity principle, loosely following Goble (2013), p. 281, we interpret the notion of specificity as entailment in the presence of (global) propositional assumptions. I.e., given a set $\mathfrak{F}$ of propositional facts about the world we say that proposition $A$ is at least as specific as proposition $B$, if $\mathfrak{F}$ entails $A \rightarrow B$. Given this interpretation, the specificity principle can be understood as limiting monotonicity of the operator $\mathcal{O}$ in the second argument in the following sense. Given a list $\mathfrak{L}$ of non-nested prima-facie obligations, and a proposition $B$, we should be licensed to infer the actual obligation $\mathcal{O}(A / B)$ if

(A) there is an injunction $\mathcal{O}_{\mathrm{pf}}(A / D)$ in $\mathfrak{L}$ which is applicable i.e. we can infer using $\mathfrak{F}$ that $B \rightarrow D$, and there is no $\mathcal{O}_{\mathrm{pf}}(X / Y)$ in $\mathfrak{L}$ such that $B$ is at least as specific 
as $Y, Y$ is at least as specific as $D$, and the formulae $A$ and $X$ are inconsistent, i.e. we can infer $\neg(A \wedge X)$.

However, while this implements the notion that more specific śrauta obligations overrule less specific conflicting ones, this only resolves conflicts between propositions $\mathcal{O}_{\text {pf }}(G / H)$ and $\mathcal{O}_{\mathrm{pf}}(I / J)$ in $\mathfrak{L}$ for which the conditions are comparable in the sense that either $H$ implies $J$ or $J$ implies $H$. Hence, to make the resulting theory consistent with MD, following the Mīmāmsā reasoning in Example 1 we add a further condition stating that

(B) there is no obligation $\mathcal{O}_{\mathrm{pf}}(X / Y) \in \mathfrak{L}$ such that $B$ is at least as specific as $Y$, the enjoined $A$ and $X$ are inconsistent, and which is not overruled by a more specific obligation $\mathcal{O}_{\mathrm{pf}}(E / F)$ from $\mathfrak{L}$.

At this point, in order to make this intuition formally precise we need to take a fundamental design decision: given that our logic MD includes monotonicity in the first argument, whenever we can derive an obligation $\mathcal{O}(A / B)$, we should also be able to derive the obligation $\mathcal{O}(A \vee C / B)$ as well. Given a list of prima-facie obligations, the question then essentially is whether we first eliminate all the conflicts from this list, and then saturate under monotonicity in the first argument (as, e.g., in the suggested procedure of removing conflicts from NDSICs in Libal and Pascucci 2019), or we first consider all the consequences of the original list under monotonicity, and then eliminate all the obligations which would yield a conflict. We clarify this with the following example.

Example 2 Consider the list containing exactly the two prima-facie obligations $\mathcal{O}_{\mathrm{pf}}(A \wedge B / C)$ and $\mathcal{O}_{\mathrm{pf}}(\neg A / C)$. Since $A \wedge B$ and $\neg A$ are inconsistent, the approach of ruling out conflicting obligations first and then saturating under monotonicity in the first argument would yield an empty set of obligations. In the second approach, instead, we first saturate under monotonicity, giving, e.g., the obligation $\mathcal{O}((A \wedge B) \vee \neg A / C)$, and only then rule out conflicts. Since $(A \wedge B) \vee \neg A$ is contradicting neither $A \wedge B$ nor $\neg A$, we thus would keep the obligation $\mathcal{O}((A \wedge B) \vee \neg A / C)$. Also, if we added the prima facie obligation $\mathcal{O}_{\mathrm{pf}}(\neg A / C \wedge D)$ we would get both $\mathcal{O}(B / C \wedge D)$ and $\mathcal{O}(\neg A / C \wedge D)$, in contrast with the first approach which would first eliminate the assumption $\mathcal{O}_{\mathrm{pf}}(A \wedge B / C)$ and hence would not yield $\mathcal{O}(B / C \wedge D)$.

While both approaches have their uses, in this work we choose to follow the second one, because of two main reasons. It allows us to preserve the power of deontic assumptions (Śrauta obligations) as much as possible, by suspending only the part of an obligation which is in conflict with another one. Moreover, it naturally implements the Mīmāmsā principle of vikalpa. Such a principle corresponds to the disjunctive response: given two incompatible prima-facie obligations $\mathcal{O}_{\mathrm{pf}}(A / B)$ and $\mathcal{O}_{\mathrm{pf}}(C / D)$, in a situation where both apply, i.e., where $B \wedge D$ holds, one may choose which one to follow, corresponding to the obligation $\mathcal{O}(A \vee C / B \wedge D)$. We now make this formally precise.

In the remainder of this paper we assume that $\mathfrak{F}$ is a finite set of sequents containing only propositional variables, which is closed under cuts, i.e., whenever $\Gamma \Rightarrow \Delta, p$ and $p, \Sigma \Rightarrow \Pi$ are in $\mathfrak{F}$, then so is $\Gamma, \Sigma \Rightarrow \Delta, \Pi$, and closed under contractions, i.e., whenever $\Gamma, p, p \Rightarrow \Delta$ or $\Gamma \Rightarrow p, p, \Delta$ are in $\mathfrak{F}$, then so are $\Gamma, p \Rightarrow \Delta$ and 

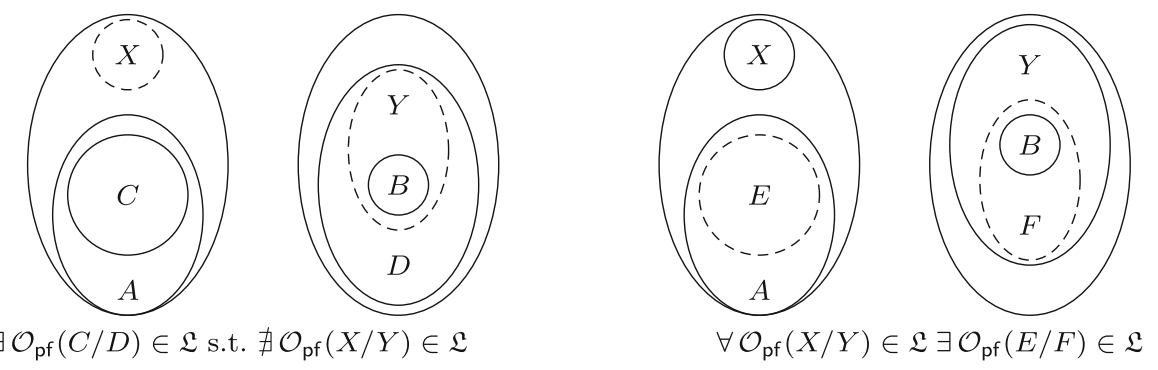

Fig. 4 A graphical representation of the conditions for $\mathcal{O}(A / B)$ being derivable. Areas can be taken as formulae with containment representing entailment, i.e., more specific formulae are contained in less specific ones

$\Gamma \Rightarrow p, \Delta$ respectively. We call $\mathfrak{F}$ the set of (propositional) facts. Note that, since every propositional formula is equivalent to a formula in conjunctive normal form and sequents containing only propositional variables correspond to clauses of a formula in conjunctive normal form, using this definition we can stipulate arbitrary propositional formulae as facts. We further assume a finite set $\mathfrak{L}$ of non-nested deontic assumptions, i.e., a finite set $\mathfrak{L}$ of formulae of the form $\mathcal{O}_{\text {pf }}(A / B)$ where $A$ and $B$ do not contain the $\mathcal{O}$-operator. We call these formulae prima-facie obligations.

Remark 4 The facts expressed by the sequnts in $\mathfrak{F}$ are assumed to be true statements about the world: we do not consider the reliability of information conveyed by such statements. Indeed, in contrast with Nute (1997), we do not distinguish actual obligations - in force under conditions that are actually verified-from apparent obligations, which are in force given all we know about morally relevant circumstances, that is under conditions that are not necessarily verified, but only believed to be true.

To capture the intuition for the specificity principle given above in a well-behaved sequent system, we first need to make the notion of implication used there formally precise. In particular, we would like to define a notion of inference $\vdash$ from the facts in $\mathfrak{F}$ depending on the set $\mathfrak{L}$, such that we can derive a sequent $\Rightarrow \mathcal{O}(A / B)$ if and only if both of the following hold, corresponding to the conditions (A) and (B) above:

- there is $\mathcal{O}_{\text {pf }}(C / D) \in \mathfrak{L}$ such that $\mathfrak{F} \vdash B \Rightarrow D$ and $\mathfrak{F} \vdash C \Rightarrow A$ and for all $\mathcal{O}_{\mathrm{pf}}(X / Y) \in \mathfrak{L}$ we have: $(\mathfrak{F} \nvdash B \Rightarrow Y$ or $\mathfrak{F} \nvdash Y \Rightarrow D$ or $\mathfrak{F} \nvdash X, A \Rightarrow)$

- for all $\mathcal{O}_{\mathrm{pf}}(X / Y) \in \mathfrak{L}$ we have: $\mathfrak{F} \nvdash B \Rightarrow Y$ or $\mathfrak{F} \nvdash X, A \Rightarrow$ or there is a $\mathcal{O}_{\text {pf }}(E / F) \in \mathfrak{L}$ such that: $(\mathfrak{F} \vdash B \Rightarrow F$ and $\mathfrak{F} \vdash F \Rightarrow Y$ and $\mathfrak{F} \vdash E \Rightarrow A)$.

To ensure that these conditions hold, we will simply turn them into premisses of the corresponding sequent rules. At first this might seem rather problematic, because we use underivability $(\nvdash)$ to define derivability $(\vdash)$. However, we will show below that the resulting notion of derivability is well-defined, using the technical tool of the cut elimination result.

Graphically, these two conditions can be visualised as in Fig. 4.

The first condition requires that the derivable obligation $\mathcal{O}(A / B)$ is implied (via upward monotonicity on the first argument and downward monotonicity on the second argument) by a less specific deontic assumption $\mathcal{O}_{\text {pf }}(C / D) \in \mathfrak{L}$ and that there is 
no $\mathcal{O}_{\text {pf }}(X / Y) \in \mathfrak{L}$ conflicting with $\mathcal{O}(A / B)$ which is more general than that and more specific than $\mathcal{O}_{\mathrm{pf}}(C / D)$. This condition includes the choice mentioned above. Indeed, it requires that any $\mathcal{O}_{\text {pf }}(X / Y) \in \mathfrak{L}$, which is more specific than $\mathcal{O}_{\text {pf }}(C / D)$ and more general than $\mathcal{O}(A / B)$, does not conflict with $\mathcal{O}(A / B)$, instead of requiring that it does not conflict with $\mathcal{O}_{\mathrm{pf}}(C / D)$. This means that the specificity principle is applied for resolving conflicts only after saturating the set of deontic assumptions under monotonicity.

The second condition models the fact that the conflicting prima-facie obligation $\mathcal{O}_{\mathrm{pf}}(X / Y)$ is overruled by the more specific prima-facie obligation $\mathcal{O}_{\mathrm{pf}}(E / F)$ by stating that $\mathfrak{F} \vdash E \Rightarrow A$, i.e., that what is enjoined by $\mathcal{O}_{\mathrm{pf}}(E / F)$ implies $A$. While this implies that $E$ and $X$ are inconsistent, one may wonder whether it is a too strong condition. In fact, as a consequence of our fundamental design decision to first saturate the set of prima-facie obligations under monotonicity, and then ruling out conflicts, the obvious alternative of only demanding $X$ and $E$ to be inconsistent would lead to conflicting obligations rather quickly. Conceptually, this is due to the fact that the more specific obligation $\mathcal{O}_{\mathrm{pf}}(E / F)$ only suspends the part of the obligation $\mathcal{O}_{\mathrm{pf}}(X / Y)$ which is in conflict with $E$, but does not cancel the obligation completely. So in particular, if this part is unrelated to $A$, then the part of $\mathcal{O}_{\mathrm{pf}}(X / Y)$ which conflicts with $\mathcal{O}(A / B)$ will remain unsuspended, and hence we should not be able to derive the latter. For example, given the list $\mathfrak{L}=\left\{\mathcal{O}_{\mathrm{pf}}(\neg p / s), \mathcal{O}_{\mathrm{pf}}(p \wedge q / s), \mathcal{O}_{\mathrm{pf}}(\neg q \wedge r / t), \mathcal{O}_{\mathrm{pf}}(\neg r / t)\right\}$, we would end up with the problematic situation of deriving both $\mathcal{O}(q / s \wedge t)$, using $\mathcal{O}_{\text {pf }}(p \wedge q / s)$ as the main obligation, and $\mathcal{O}(\neg q / s \wedge t)$, using $\mathcal{O}_{\text {pf }}(\neg q \wedge r / t)$ as the main obligation. The stronger condition used above prevents this situation.

To turn these considerations into sequent rules (the rules $\mathcal{O}_{L}^{\mathcal{O}_{\mathrm{pf}}(C / D)}, \mathcal{O}_{R}^{\mathcal{O}_{\mathrm{pf}}(C / D)}$ in Definition 1 below), we convert every (meta-)conjunction and universal quantifier in this characterization into different premises, while (meta-)disjunctions and existential quantifiers yield a split into different rules. To write the rules in an economic way, we use the following notation.

Notation 1 If $\mathcal{P}$ is a set of premisses, and $S=\left\{\mathcal{S}_{1}, \ldots, \mathcal{S}_{n}\right\}$ is a set of sets of premisses we write

$$
\frac{\mathcal{P} \cup[S]}{C} \text { for the set of rules }\left\{\frac{\mathcal{P} \cup \mathcal{S}_{1}}{C}, \ldots, \frac{\mathcal{P} \cup \mathcal{S}_{n}}{C}\right\}
$$

e.g., we write

$$
\frac{\{X \Rightarrow Y\} \cup\left[\left\{\{\Sigma \Rightarrow \Pi\},\left\{\Omega \Rightarrow \Theta, A_{i} \mid A_{i} \in \mathcal{F}\right\}\right\}\right]}{\Gamma \Rightarrow \Delta}
$$

for the set containing the two rules

$$
\frac{\{X \Rightarrow Y\} \cup\{\Sigma \Rightarrow \Pi\}}{\Gamma \Rightarrow \Delta} \quad \text { and } \quad \frac{\{X \Rightarrow Y\} \cup\left\{\Omega \Rightarrow \Theta, A_{i} \mid A_{i} \in \mathcal{F}\right\}}{\Gamma \Rightarrow \Delta} .
$$

Note that we use set-theoretic notation for the sets of premisses, e.g., the rule above left has the two premisses $X \Rightarrow Y$ and $\Sigma \Rightarrow \Pi$ and the conclusion $C$. 
Since the rules now also will mention underivability, we further need to add a judgment for this to some of the sequents, written as $(\mathfrak{F}, \mathfrak{L}) \nvdash_{\mathrm{G}_{M D} \text { cut }}$, with the intended meaning that the sequent is not derivable from the facts $\mathfrak{F}$ and the prima-facie deontic statements $\mathfrak{L}$ in the system $G_{M D C u t}$ in the sense defined below (Definition 2). Thus we will obtain a set of rules $\mathcal{O}_{R}^{\mathcal{O}_{\text {pf }}(C / D)}$ introducing a formula of the form $\mathcal{O}(A / B)$ on the right hand side of the sequent. For technical reasons we will also add rules $\mathcal{O}_{L}^{\mathcal{O}_{\mathrm{pf}}(C / D)}$ introducing such a formula on the left hand side-these essentially follow from absorbing inferences using the axiom (D) into the previous rule. We will show below, in the discussion of the full system, that their addition indeed is merely a technical convenience (see Lemma 1).

Remark 5 The formulae we want to infer might have nested deontic operators, setting the system apart from, e.g., the known systems of Input/Output logic (Makinson and van der Torre 2000). Indeed, they should capture key prescriptions like "under the condition of having to perform sacrifice $\alpha$ under the conditions $\beta$, you ought to do $\gamma$ ". However, to ensure decidability of the system we do not permit nested obligations in the deontic assumptions.

Definition 1 Let $\mathfrak{L}=\left\{\mathcal{O}_{\mathrm{pf}}\left(A_{1} / B_{1}\right), \ldots, \mathcal{O}_{\mathrm{pf}}\left(A_{n} / B_{n}\right)\right\}$ be a finite set of non-nested prima-facie obligation formulae and let $\mathfrak{F}$ be a set of propositional sequents. The rules of $_{\mathrm{ga}_{\mathfrak{L}}}$ (for global assumptions from $\mathfrak{L}$ ) are given in Fig. 5. A proto-derivation with conclusion $\Gamma \Rightarrow \Delta$ in the system $\mathrm{G}_{\mathrm{MD}}$ from assumptions $(\mathfrak{F}, \mathfrak{L})$ is a finite labelled tree, where each internal node is labelled with a sequent, each leaf is labelled with an initial sequent, a sequent from $\mathfrak{F}$, or an underivability statement $(\mathfrak{F}, \mathfrak{L}) \nvdash_{\mathrm{G}_{\mathrm{MD}} \text { cut }} \Sigma \Rightarrow \Pi$, such that the label of every internal node is obtained from the labels of its children using the rules of $\mathrm{G}_{\mathrm{MD}}$ or $\mathrm{ga} \mathrm{a}_{\mathfrak{L}}$. The notion of a proto-derivation in the system $\mathrm{G}_{\mathrm{MD}} \mathrm{cut}$ is defined analogously, but also permitting applications of the cut rule

$$
\frac{\Gamma \Rightarrow \Delta, A \quad A, \Sigma \Rightarrow \Pi}{\Gamma, \Sigma \Rightarrow \Delta, \Pi} \text { cut }
$$

The depth of a proto-derivation is the depth of the underlying tree, i.e., the maximal length of a branch in the tree plus one.

For future reference we divide the premisses of the rules in Fig. 5 into different blocks in the following way: the first two premisses, i.e., $\{B \Rightarrow D\}$ and $\{C \Rightarrow A\}$ together form the standard block, stating that the prima-facie obligation $\mathcal{O}_{\mathrm{pf}}(C / D)$ potentially can be used to derive the conclusion $\mathcal{O}(A / B)$. The following block

$$
\left\{\left[\begin{array}{l}
\left\{(\mathfrak{F}, \mathfrak{L}) \nvdash_{\mathrm{G}_{\mathrm{MD}} \text { cut }} B \Rightarrow Y\right\} \\
\left.(\mathfrak{F}, \mathfrak{L}) \nvdash_{\mathrm{G}_{\mathrm{MD}} \text { cut }} Y \Rightarrow D\right\} \\
\left.(\mathfrak{F}, \mathfrak{L}) \nvdash_{\mathrm{G}_{\mathrm{MD}} \text { cut }} X, A \Rightarrow\right\}
\end{array}\right] \mid \mathcal{O}_{\mathrm{pf}}(X / Y) \in \mathfrak{L}\right\}
$$

is called the not-excepted block, and states that the prima-facie obligation $\mathcal{O}_{\mathrm{pf}}(C / D)$ is not overruled by another one which is at least as specific. The remaining premisses together form the no-active-conflict block, which states that there is no other conflicting 


$$
\begin{aligned}
& \{B \Rightarrow D\} \quad \cup \quad\{C \Rightarrow A\} \\
& \cup\left\{\left[\begin{array}{l}
\left\{\left\{(\mathfrak{F}, \mathfrak{L}) \nvdash \nvdash_{\mathrm{G}_{\mathrm{MD}} \text { cut }} B \Rightarrow Y\right\}\right\} \\
\cup\left\{(\mathfrak{F}, \mathfrak{L}) \nvdash_{\mathrm{G}_{\mathrm{MD}} \text { cut }} Y \Rightarrow D\right\} \\
\cup\left\{\left\{(\mathfrak{F}, \mathfrak{L}) \nvdash \nvdash_{\mathrm{G}_{\mathrm{MD}} \text { cut }} X, A \Rightarrow\right\}\right\}
\end{array}\right] \mid \mathcal{O}_{\mathrm{pf}}(X / Y) \in \mathfrak{L}\right\}
\end{aligned}
$$

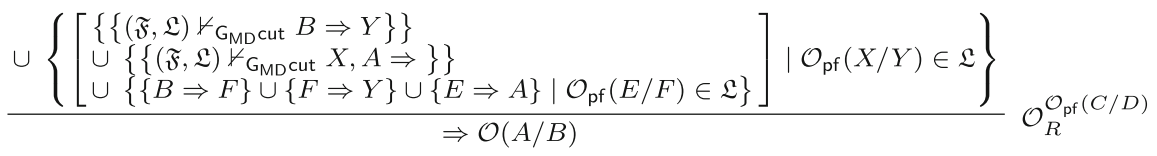

$$
\begin{aligned}
& \{B \Rightarrow D\} \quad \cup \quad\{C, A \Rightarrow\} \\
& \cup\left\{\left[\begin{array}{l}
\left\{\left\{(\mathfrak{F}, \mathfrak{L}) \nvdash_{\mathrm{G}_{\mathrm{MD}} \text { cut }} B \Rightarrow Y\right\}\right\} \\
\cup\left\{\left\{(\mathfrak{F}, \mathfrak{L}) \nvdash_{\mathrm{G}_{\mathrm{MD}} \text { cut }} Y \Rightarrow D\right\}\right. \\
\left.\cup\left\{\left\{(\mathfrak{F}, \mathfrak{L}) \nvdash_{\mathrm{G}_{\mathrm{MD}} \text { cut }} X \Rightarrow A\right)\right\}\right\}
\end{array}\right] \mid \mathcal{O}_{\mathrm{pf}}(X / Y) \in \mathfrak{L}\right\}
\end{aligned}
$$

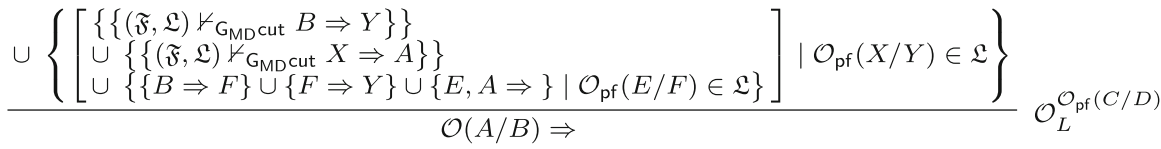

Fig. 5 The rules of ga $\mathfrak{L}$ with $\mathcal{O}_{\text {pf }}(C / D) \in \mathfrak{L}$

prima-facie obligation which is not overruled by a more specific one. For every formula $\mathcal{O}_{\mathrm{pf}}(X / Y)$ the choices are divided into the no-conflict block, stating that there is no conflict between the prima-facie obligation $\mathcal{O}_{\mathrm{pf}}(X / Y)$ and the desired conclusion $\mathcal{O}(A / B)$, and consisting of the underivability statements

$$
(\mathfrak{F}, \mathfrak{L}) \nvdash_{\mathrm{G}_{\mathrm{MD}} \text { cut }} B \Rightarrow Y \quad \text { and } \quad(\mathfrak{F}, \mathfrak{L}) \nvdash_{\mathrm{G}_{\mathrm{MD}} \text { cut }} X, A \Rightarrow \text {, }
$$

and the override block, consisting of the remaining possible premisses

$$
\left\{\{B \Rightarrow F\} \cup\{F \Rightarrow Y\} \cup\{E, A \Rightarrow\} \mid \mathcal{O}_{\mathrm{pf}}(E, F) \in \mathfrak{L}\right\}
$$

and stating that the prima-facie obligation $\mathcal{O}_{\mathrm{pf}}(X / Y)$ is overruled by another one which is at least as specific. The terminology for the rule $\mathcal{O}_{L}^{\mathcal{O}_{\text {pf }}(C / D)}$ is analogous.

Definition 2 A proto-derivation in $G_{M D}$ (in $G_{M D}$ cut) from $(\mathfrak{F}, \mathfrak{L})$ is valid if for each of the underivability statements $(\mathfrak{F}, \mathfrak{L}) \nvdash_{\mathrm{G}_{\mathrm{MD}} \text { cut }} \Sigma \Rightarrow \Pi$, occurring as one of the leafs of that derivation, there is no valid proto-derivation of $\Sigma \Rightarrow \Pi$ in $\mathrm{G}_{\mathrm{MD}}$ cut from $(\mathfrak{F}, \mathfrak{L})$. In case there is such a valid proto-derivation we also write $(\mathfrak{F}, \mathfrak{L}) \vdash_{\mathrm{G}_{\mathrm{MD}}} \Gamma \Rightarrow \Delta$ and $(\mathfrak{F}, \mathfrak{L}) \vdash_{\mathrm{G}_{\mathrm{MD}} \text { cut }} \Gamma \Rightarrow \Delta$ respectively.

Note that underivability statements are always evaluated in the system with the cut rule. Since the definition of a valid proto-derivation involves the notion of a valid proto-derivation itself, it is not immediately clear that this notion is well-defined. We will show in the discussion of the full system below (Corollary 1) that this is indeed the case. In particular, this along with the decidability result follows from the crucial cut elimination theorem, stating the redundancy of the cut rule: 


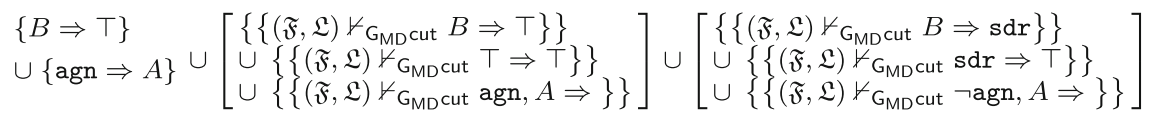

$$
\begin{aligned}
& \cup\left[\begin{array}{l}
\left\{\left\{(\mathfrak{F}, \mathfrak{L}) \nvdash_{\mathrm{G}_{\mathrm{MD}} \text { cut }} B \Rightarrow \top\right\}\right\} \\
\cup\left\{\left\{(\mathfrak{F}, \mathfrak{L}) \nvdash_{\mathrm{G}_{\mathrm{MD}} \text { cut }} \text { agn, } A \Rightarrow\right\}\right\} \\
\cup\{\{B \Rightarrow T\} \cup\{T \Rightarrow \top\} \cup\{\text { agn } \Rightarrow A\}\} \\
\cup\{\{B \Rightarrow \mathrm{sdr}\} \cup\{\mathrm{sdr} \Rightarrow \top\} \cup\{\neg \text { agn } \Rightarrow A\}\}
\end{array}\right]
\end{aligned}
$$

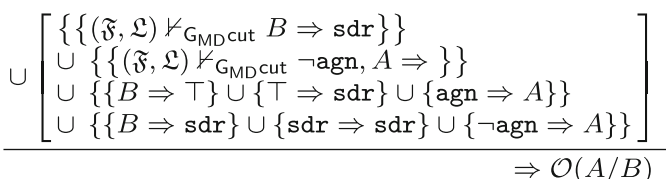

Fig. 6 The rule $\mathcal{O}_{R}^{\mathcal{O}_{\mathrm{pf}}(C / D)}$ from Example 3

Proposition 2 (Ciabattoni et al. 2018) For every $\mathfrak{F}, \mathfrak{L}$ and sequent $\Gamma \Rightarrow \Delta$ we have

$$
(\mathfrak{F}, \mathfrak{L}) \vdash_{G_{M D} \text { cut }} \Gamma \Rightarrow \Delta \quad \text { if and only if } \quad(\mathfrak{F}, \mathfrak{L}) \vdash_{G_{M D}} \Gamma \Rightarrow \Delta
$$

Since this proposition is a special case of the more general result for the full system in Theorem 2 below we omit the proof.

Example 3 Consider the prima-facie obligations given by $\mathfrak{L}=\left\{\mathcal{O}_{\mathrm{pf}}(\operatorname{agn} / \top)\right.$, $\left.\mathcal{O}_{\text {pf }}(\neg \operatorname{agn} / \mathrm{sdr})\right\}$ (with agn, sdr and tch as in Example 1) and the set $\mathfrak{F}=\varnothing$ of facts. Taking the formula $\mathcal{O}_{\mathrm{pf}}(\mathrm{agn} / \top)$ as the formula $\mathcal{O}_{\mathrm{pf}}(C / D)$ in the general scheme of Fig. 5, we obtain the rules in Fig. 6.

In particular, the sequent $\Rightarrow \mathcal{O}($ agn/tch) would be derivable using, e.g., an instance of the rule

$$
\begin{aligned}
& B \Rightarrow \top \text { agn } \Rightarrow A \quad(\mathfrak{F}, \mathfrak{L}) \nvdash_{\mathrm{G}_{\mathrm{MD}} \text { cut }} \text { agn, } A \Rightarrow \quad(\mathfrak{F}, \mathfrak{L}) \nvdash_{\mathrm{G}_{\mathrm{MD}} \text { cut }} B \Rightarrow \text { sdr } \\
& B \Rightarrow \top \quad \top \Rightarrow \top \text { agn } \Rightarrow A \quad(\mathfrak{F}, \mathfrak{L}) \nvdash_{\mathrm{G}_{\mathrm{MD}} \text { cut }} B \Rightarrow \mathrm{sdr} \\
& \Rightarrow \mathcal{O}(A / B)
\end{aligned}
$$

Similarly, taking the formula $\mathcal{O}_{\mathrm{pf}}(C / D)$ to be $\mathcal{O}_{\mathrm{pf}}(\neg \mathrm{agn} / \mathrm{sdr})$ we obtain, e.g.

$$
\begin{gathered}
B \Rightarrow \operatorname{sdr} \neg \operatorname{agn} \Rightarrow A \quad(\mathfrak{F}, \mathfrak{L}) \nvdash_{\mathrm{G}_{\mathrm{MD}} \mathrm{cut}} \top \Rightarrow \operatorname{sdr} \quad(\mathfrak{F}, \mathfrak{L}) \nvdash_{\mathrm{G}_{\mathrm{MD}} \text { cut }} \neg \text { agn }, A \Rightarrow \\
B \Rightarrow \operatorname{sdr} \operatorname{sdr} \Rightarrow \top \quad \neg \operatorname{agn} \Rightarrow A \quad B \Rightarrow \operatorname{sdr} \quad \operatorname{sdr} \Rightarrow \operatorname{sdr} \neg \text { agn } \Rightarrow A
\end{gathered}
$$

which serves to derive the sequent $\Rightarrow \mathcal{O}(\neg \operatorname{agn} / \operatorname{tch} \wedge$ sdr $)$. Finally, using ga ${ }_{L}$ with $\mathcal{O}_{\mathrm{pf}}(\neg \mathrm{agn} / \mathrm{sdr})$ for the formula $\mathcal{O}_{\mathrm{pf}}(C / D)$ yields a derivation of $\mathcal{O}(\mathrm{agn} / \mathrm{tch} \wedge$ $\mathrm{sdr}) \Rightarrow$ and thus $\Rightarrow \neg \mathcal{O}(\operatorname{agn} / \operatorname{tch} \wedge \mathrm{sdr})$. Note that even for just two prima-facie obligations we obtain many (often redundant) rules. 


\section{Extending bMDL with new deontic operators}

The preliminary analysis of Mīmāmsā reasoning purely in terms of obligations is rather simplistic, since it considers other deontic concepts such as prohibitions as defined notions. It turned out, indeed, that obligations and prohibitions are treated markedly different ${ }^{3}$ in Mīmāmsāa : on a "meta-logical" level, obeying a Vedic obligation gives positive results and disrespecting it implies just the lack of these results; conversely, the observance of a Vedic prohibition gives no result and the violation of it leads to a sanction (the accumulation of negative karma). Hence it is not enough to model prohibitions as negative obligations.

In addition the difference between prescriptions and prohibitions is not only on the results of obeying or disrespecting them; one of the most important differences is the idea at the base of those two deontic concepts, i.e. "activation" in case of injunctions and "inhibition" for prohibitions. To confirm such a distinction, let us consider the debate in Mīmāmsāa commentaries on the injunction not to eat kalajañja (probably a variety of garlic). If the command represents a prohibition, it means that, independently from the agents' desires and motivations, the agents have the duty not to consume this product: in principle, even eating it by accident would constitute a violation. On the other hand, if the command is a negative obligation, the agents have the duty to choose to refrain from eating kalañja; hence, theoretically, if the agents decide to eat that vegetable, they are not compliant with the obligation, even if an external contingency prevents them from realizing their intentions. For these reasons, prescriptions and prohibitions should be considered as genuine deontic concepts: they cannot be deprived of their deontic content and reduced to instructions for obtaining desirable results or avoiding sanctions. Such an interpretation-that could be formally represented by a KangerAndersonian reduction-would be closer to the instrumental reading of commands given by the late author Mandana Miśra (c. 8th century CE), which was in between the Mīmāmsāa and the Vedanta schools of Indian philosophy.

In this section we continue and refine the analysis of Mīmāmsā reasoning in Ciabattoni et al. (2015) by extending the $\square$-free fragment of the logic bMDL with new operators for prohibitions and recommendations. We call the resulting logic MD+. As for the obligation operator $\mathcal{O}$, axioms and rules for the new operators are extracted from the Mìmāmsā nyāyas, that have been in the meanwhile ${ }^{4}$ found in the texts, translated from Sanskrit, interpreted and abstracted.

Prohibitions are modeled in MD+ using the operator $\mathcal{F}(A / B)$, to be read as " $A$ is forbidden under the conditions $B$ ". As in the case of obligations, prohibitions are better expressed by a dyadic operator. They can apply unconditionally to the person throughout her life (purusārtha), as in the Vedic command "one should not perform violence on any living being", or be relative to a particular ritual context (kratvartha), as for the example "one should not utter the 'ye yajāmahe' mantra during the aftersacrifices” discussed in Jaimini’s Pūrva Mìmāmsā Sūtra (henceforth PMS).

\footnotetext{
3 A similar phenomenon happens in Talmudic logic, were distinct operators are needed (Abraham et al. 2011).

4 This is an ongoing project, which is carried out together with Sanskritists.
} 
A first natural property for prohibitions is expressed by the axiom $D_{\mathcal{F}}$

$$
\neg(\mathcal{F}(A / B) \wedge \mathcal{F}(\neg A / B))
$$

motivated by the consideration that, because of the "meaningfulness of Vedic commands" (in PMS 1.2.23) stating that no injunction can be meaningless or inapplicable, there is always a way to obey all needed commands and avoid sanctions; this would be impossible having the prohibition of an action and its negation under the same conditions. The downward monotonicity rule $\left(\operatorname{Mon}_{\mathcal{F}}\right)$

$$
\frac{C \rightarrow A \quad B \leftrightarrow D}{\mathcal{F}(A / B) \rightarrow \mathcal{F}(C / D)}
$$

is justified by argumentations as the one in Medhātithi's Manubhāṣya, where the prohibition to commit suicide $(\mathcal{F}$ (suicide $/ \top))$ is derived from the more comprehensive prohibition to commit violence $(\mathcal{F}$ (violence/ $\top))$, since there it is explicitly assumed that suicide $\rightarrow$ kill and kill $\rightarrow$ violence.

Finally, the axiom $D_{\mathcal{O F}}$

$$
\neg(\mathcal{O}(A / B) \wedge \mathcal{F}(A / B))
$$

arises again from the nyāya about meaningfulness of commands, and from what is known as "ought implies can principle", extracted from the discussion on the concept of adhikāra in Jaimini's texts. According to this principle, a person who is prescribed to perform an action is assumed to have not only physical and economical capacities, but also the "practical" possibility to complete the action without undesirable consequences, like damages or sanctions. Therefore, performing a prescribed act can never lead to a sanction, hence it is impossible that the same act is prohibited.

Recommendations Besides the distinction between obligations and prohibitions, a more refined analysis should take into account the different notions of prescriptions used by Mīmāmsāā authors. Traditionally, rituals prescribed by the Vedas are distinguished into fixed (nitya), occasional (naimittika), and elective (kāmya); fixed ritual actions should be performed, in order to obtain the positive result of good karma, regularly throughout the whole life, occasional ones have similar properties, but should be carried out in special occasions, like the birth of a child, whilst the third kind includes rituals to be executed only in order to obtain a specific result. It has been noticed (Freschi et al. 2019) that, while the characteristics of the operator $\mathcal{O}$ in bMDL are well suited to describe fixed and occasional prescriptions, the elective rituals represent recommendations or instruction for achieving a result in a "Vedic" way, more than proper obligations. We call these weaker obligations recommendations and express them with the operator $\mathcal{R}(. /$.). We model $\mathcal{R}(. /$.) using the dyadic version of the modal logic MP, see, e.g., Chellas (1980), in line with the analysis in Freschi et al. (2019) where also the axioms for $\mathcal{R}(. /$.) are motivated. Note that it might be possible for something to be obligatory and recommended at the same time, as, e.g., in the case of the Agnihotra sacrifice. Indeed, the sequence of actions constituting the Agnihotra ritual represents the content both of a fixed sacrifice (corresponding to obligations) and of an elective 


$$
\begin{aligned}
& \left(\operatorname{Mon}_{\mathcal{F}}\right): \frac{C \rightarrow A \quad B \leftrightarrow D}{\mathcal{F}(A / B) \rightarrow \mathcal{F}(C / D)} \quad\left(\operatorname{Mon}_{\mathcal{R}}\right): \frac{A \rightarrow C \quad B \leftrightarrow D}{\mathcal{R}(A / B) \rightarrow \mathcal{R}(C / D)} \\
& \left(\mathrm{D}_{\mathcal{F}}\right): \quad \neg(\mathcal{F}(A / B) \wedge \mathcal{F}(\neg A / B)) \quad\left(\mathrm{P}_{\mathcal{R}}\right): \quad \neg \mathcal{R}(\perp / A) \\
& \left(\mathrm{D}_{\mathcal{O F}}\right): \quad \neg(\mathcal{O}(A / B) \wedge \mathcal{F}(A / B))
\end{aligned}
$$

Fig. 7 The Hilbert-style axiomatisations for prohibitions and weak obligations in MD+

one (corresponding to recommendations). In other words, if the agents perform such a ritual perfectly, according to the stricter rules governing elective sacrifices, they are compliant both with the recommendation (hence obtaining the desired result) and with the obligation (fulfilling their duties).

The axiom

$$
\neg \mathcal{R}(\perp / A)
$$

guarantees that there are no self-contradictory recommendations, which represents a minimal condition for any Vedic instruction. Notice that it is weaker than $\mathrm{D}_{\mathcal{O}}$ and $D_{\mathcal{F}}$, as, in contrast with obligations and prohibitions, it is possible to have two recommended rituals for getting the same result which cannot be performed at the same time. In those cases (e.g., in the case of the prescriptions of kāriri sacrifice and twelvenights sacrifice for obtaining the rain, see, e.g., Freschi et al. 2019) Mīmāmsāa authors assume that one of the two sacrifices is enough to get the intended result, but both recommendations remain in force.

The rule

$$
\frac{A \rightarrow C \quad B \leftrightarrow D}{\mathcal{R}(A / B) \rightarrow \mathcal{R}(C / D)}
$$

is justified by the following abstraction of the nyāyas in the Tantrarahasya IV.4.3.3 (see Freschi 2012):

if the accomplishment of X presupposes the accomplishment of $\mathrm{Y}$, the obligation to perform $\mathrm{X}$ prescribes also $\mathrm{Y}$.

Already mentioned in Ciabattoni et al. (2015) regarding obligations, this principle is suitable also for recommendations because, as noticed in Freschi et al. (2019), it is more about how Mīmāmsāa authors consider the relations among facts than about a specific kind of prescription.

The axioms and rules of all the operators in a Hilbert-style system are given in Fig. 7, and the corresponding sequent rules, obtained using the method in Lellmann and Pattinson (2013), are given in Fig. 8. Note that the resulting sequent calculus admits cut-elimination by construction and hence the resulting logic is consistent.

Permissions MD+ does not include an explicit operator for permissions: they are instead treated exclusively as explicit exceptions to obligations or to prohibitions, and hence considered only on the prima-facie level. This formalization is motivated by Mīmāṃsā authors' interpretation, which assumes that "there cannot be a prescription prescribing a person to do something she is already inclined to do" (novelty nyāya in 


$$
\begin{aligned}
& \frac{C \Rightarrow A \quad B \Rightarrow D \quad D \Rightarrow B}{\mathcal{F}(A / B) \Rightarrow \mathcal{F}(C / D)} \operatorname{Mon}_{\mathcal{F}} \quad \frac{A \Rightarrow C \quad B \Rightarrow D \quad D \Rightarrow B}{\mathcal{O}(A / B), \mathcal{F}(C / D) \Rightarrow} \mathrm{D}_{\mathcal{O F}} \\
& \frac{A \Rightarrow C \quad B \Rightarrow D \quad D \Rightarrow B}{\mathcal{R}(A / B) \Rightarrow \mathcal{R}(C / D)} \operatorname{Mon}_{\mathcal{R}} \quad \frac{A \Rightarrow}{\mathcal{R}(A / B) \Rightarrow} \mathrm{P}_{\mathcal{R}} \\
& \frac{\Rightarrow A, B \quad C \Rightarrow D \quad D \Rightarrow C}{\mathcal{F}(A / C), \mathcal{F}(B / D) \Rightarrow} \mathrm{D}_{\mathcal{F}} \quad \frac{\Rightarrow A}{\mathcal{F}(A / B) \Rightarrow} \mathrm{P}_{\mathcal{F}}
\end{aligned}
$$

Fig. 8 The sequent rules of $\mathrm{G}_{\mathrm{MD}+}$ for the logic with prohibitions and recommendations

$$
\frac{B \Rightarrow D \quad D \Rightarrow B \quad C \Rightarrow A \quad(\mathfrak{F}, \mathfrak{L}) \nvdash_{\mathrm{G}_{\mathrm{MD}+\text { cut }}} C \Rightarrow}{\Rightarrow \mathcal{R}(A / B)} \mathcal{R}_{R}^{\mathcal{R}_{\mathrm{pf}}(C / D)}
$$

Fig. 9 The global assumption rules for recommendations, based on the prima-facie recommendation $\mathcal{R}_{\text {pf }}(C / D) \in \mathfrak{L}$

Jaimini's PMS 1.2.19). Hence permissions, having the same linguistic form as prescriptions but conveying something that is naturally desired by anyone, are interpreted as exceptions to more general commands. For instance, the statement (in Śabara on PMS 10.7.28) "the five five-nailed animals can be eaten" is interpreted, at the primafacie level, as the prohibition to eat meat plus the permission to eat five species of five-nailed animals (i.e., some species of wild rodents, wild boars, lizards, hares, and turtles).

\section{Defeasible reasoning in Mīmāṃsā}

We introduce a sequent calculus to reason in presence of specificity in the extended logic. In order to incorporate into our framework prohibitions, permissions as exceptions to prescriptions or prohibitions, and recommendations, we extend the list of deontic assumptions or prima-facie (śrauta) prescriptions to also include primafacie prohibitions, prima-facie obligation-permissions (exceptions to obligations), prima-facie prohibition-permissions (exceptions to prohibitions) and prima-facie recommendations, denoted by the operators $\mathcal{F}_{\text {pf }}(. /),. \mathcal{P}_{\text {pf }}^{\mathcal{O}}(. /),. \mathcal{P}_{\text {pf }}^{\mathcal{F}}(. /$.$) and \mathcal{R}_{\text {pf }}(. /$. respectively. Hence, the list $\mathfrak{L}$ of prima-facie deontic statements now contains finitely many (non-nested) formulae of these forms. In particular, if $\mathfrak{L}$ only contains prima-facie obligation formulae, we recover the simplified situation of Sect. 3. The construction of the global assumption rules then follows the same principle as before, incorporating specificity.

For the recommendations we need to make sure that we do not derive $\mathcal{R}(A / B)$ with $A$ equivalent to $\perp$. In particular, following Freschi et al. (2019) we use the Mīmāmsā reasoning that the Vedas do not recommend anything which is self-contradictory, to rule out prima-facie recommendations $\mathcal{R}(A / D)$ where it follows from the facts that $A$ implies $\perp$. Hence we only need one global assumption rule of the form given in Fig. 9.

Remark 6 Due to the presence in $\mathrm{MD}+$ of axiom $\mathrm{D}_{\mathcal{O F}}$, the cases for the obligations and prohibitions are somewhat more complex than cases involving recommendations, 
as prima-facie obligations and prohibitions can overrule each others according to the specificity principle.

For the sake of an economical presentation we employ Notation 1 from Sect. 3.1. The rationale for the construction of the rules then is as follows:

- Due to $D_{\mathcal{O}}$ (resp. $D_{\mathcal{F}}$ ), more specific conflicting obligations (resp. prohibitions) overrule less specific obligations (resp. prohibitions).

- Due to the interaction rule $D_{\mathcal{O F}}$, more specific conflicting obligations overrule less specific prohibitions and vice versa.

- Due to the interpretation of permissions as explicit exceptions to obligations or prohibitions, more specific obligation-permissions overrule less specific obligations, but have no relevance for prohibitions, and analogously for prohibitionpermissions.

Right rules Following this, for obligations we obtain the following characterisation. An obligation $\mathcal{O}(A / B)$ follows from a set $\mathfrak{L}$ of śrauta deontic statements if there is a śrauta obligation $\mathcal{O}_{\mathrm{pf}}(C / D)$ in $\mathfrak{L}$ such that:

- The assumption is applicable, because the condition $B$ is at least as specific as $D$, i.e., $(\mathfrak{F}, \mathfrak{L}) \vdash B \Rightarrow D$.

- $A$ is entailed by $C$, i.e., $(\mathfrak{F}, \mathfrak{L}) \vdash C \Rightarrow A$.

- There is no more specific conflicting śrauta obligation or obligation-permission $\left(\mathcal{P}_{\text {pf }}^{\mathcal{O}}\right)$, i.e., for every $\mathcal{O}_{\text {pf }}(X / Y) \in \mathfrak{L}$ or $\mathcal{P}_{\text {pf }}^{\mathcal{O}}(X / Y) \in \mathfrak{L}$ we have $((\mathfrak{F}, \mathfrak{L}) \nvdash B \Rightarrow Y$ or $(\mathfrak{F}, \mathfrak{L}) \nvdash Y \Rightarrow D$ or $(\mathfrak{F}, \mathfrak{L}) \nvdash X, A \Rightarrow)$.

- There is no more specific conflicting śrauta prohibition, i.e., for every $\mathcal{F}_{\mathrm{pf}}(X / Y) \in$ $\mathfrak{L}$ we have $((\mathfrak{F}, \mathfrak{L}) \nvdash B \Rightarrow Y$ or $(\mathfrak{F}, \mathfrak{L}) \nvdash Y \Rightarrow D$ or $(\mathfrak{F}, \mathfrak{L}) \nvdash A \Rightarrow X)$.

- Every conflicting śrauta obligation is overruled by a more specific obligation, prohibition, or obligation-permission $\left(\mathcal{P}_{\text {pf }}^{\mathcal{O}}\right)$ i.e., for every śrauta obligation $\mathcal{O}_{\mathrm{pf}}(X / Y)$ with $(\mathfrak{F}, \mathfrak{L}) \vdash B \Rightarrow Y$ and $(\mathfrak{F}, \mathfrak{L}) \vdash X, A \Rightarrow$ there is a śrauta obligation $\mathcal{O}_{\text {pf }}(E / F)$ with $((\mathfrak{F}, \mathfrak{L}) \vdash B \Rightarrow F$ and $(\mathfrak{F}, \mathfrak{L}) \vdash F \Rightarrow Y$ and $(\mathfrak{F}, \mathfrak{L}) \vdash E \Rightarrow A$ ) or there is a śrauta prohibition $\mathcal{F}_{\text {pf }}(E / F)$ with $((\mathfrak{F}, \mathfrak{L}) \vdash B \Rightarrow F$ and $(\mathfrak{F}, \mathfrak{L}) \vdash$ $F \Rightarrow Y$ and $(\mathfrak{F}, \mathfrak{L}) \vdash \Rightarrow A, E)$ or there is a śrauta permission $\mathcal{P}_{\mathrm{pf}}^{\mathcal{O}}(E / F)$ with $((\mathfrak{F}, \mathfrak{L}) \vdash B \Rightarrow F$ and $(\mathfrak{F}, \mathfrak{L}) \vdash F \Rightarrow Y$ and $(\mathfrak{F}, \mathfrak{L}) \vdash E \Rightarrow A)$.

- Every conflicting śrauta prohibition is overruled by a more specific obligation, prohibition or prohibition-permission, i.e., for every śrauta prohibition $\mathcal{F}_{\mathrm{pf}}(X / Y)$ with $(\mathfrak{F}, \mathfrak{L}) \vdash B \Rightarrow Y$ and $(\mathfrak{F}, \mathfrak{L}) \vdash A \Rightarrow X$ there is a srauta obligation $\mathcal{O}_{\mathrm{pf}}(E / F)$ with $((\mathfrak{F}, \mathfrak{L}) \vdash B \Rightarrow F$ and $(\mathfrak{F}, \mathfrak{L}) \vdash F \Rightarrow Y$ and $(\mathfrak{F}, \mathfrak{L}) \vdash E \Rightarrow A$ ) or there is a śrauta prohibition $\mathcal{F}_{\text {pf }}(E / F)$ with $((\mathfrak{F}, \mathfrak{L}) \vdash B \Rightarrow F$ and $(\mathfrak{F}, \mathfrak{L}) \vdash F \Rightarrow Y$ and $(\mathfrak{F}, \mathfrak{L}) \vdash \Rightarrow A, E)$ or there is a śrauta permission $\mathcal{P}_{\text {pf }}^{\mathcal{F}}(E / F)$ with $((\mathfrak{F}, \mathfrak{L}) \vdash B \Rightarrow$ $F$ and $(\mathfrak{F}, \mathfrak{L}) \vdash F \Rightarrow Y$ and $(\mathfrak{F}, \mathfrak{L}) \vdash E \Rightarrow A$ ).

- Every conflicting śrauta obligation-permission is overruled by a more specific obligation, i.e., for every śrauta obligation-permission $\mathcal{P}_{\mathrm{pf}}^{\mathcal{O}}(X / Y)$ with $(\mathfrak{F}, \mathfrak{L}) \vdash$ $B \Rightarrow Y$ and $(\mathfrak{F}, \mathfrak{L}) \vdash A, X \Rightarrow$ there is a srauta obligation $\mathcal{O}_{\mathrm{pf}}(E / F)$ with $((\mathfrak{F}, \mathfrak{L}) \vdash B \Rightarrow F$ and $(\mathfrak{F}, \mathfrak{L}) \vdash F \Rightarrow Y$ and $(\mathfrak{F}, \mathfrak{L}) \vdash E \Rightarrow A)$.

The notion of being conflicting here is different, depending on the two conflicting statements. In particular, two obligations $\mathcal{O}_{\text {pf }}(A / B)$ and $\mathcal{O}_{\text {pf }}(C / D)$ are conflicting if 


$$
\begin{aligned}
& \{B \Rightarrow D\} \quad\{C \Rightarrow A\}
\end{aligned}
$$

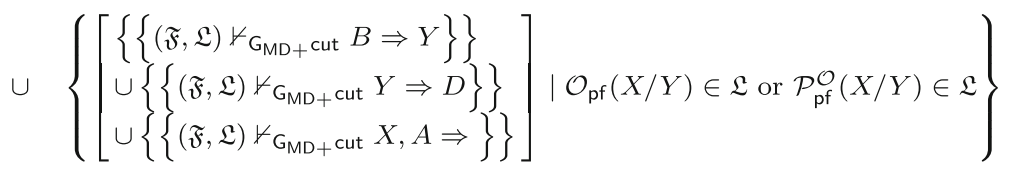

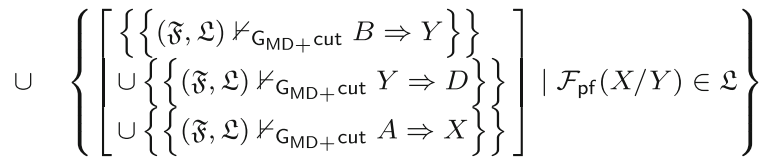

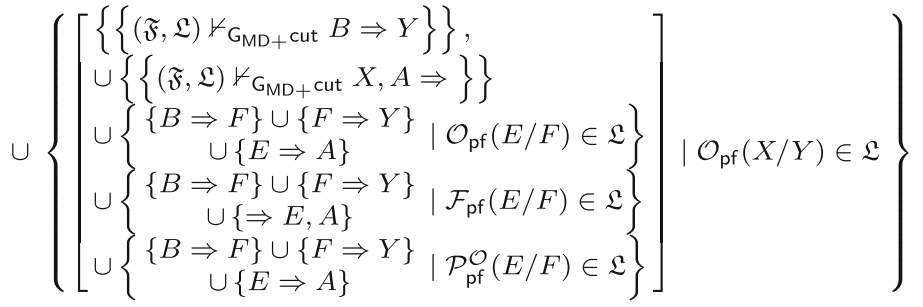

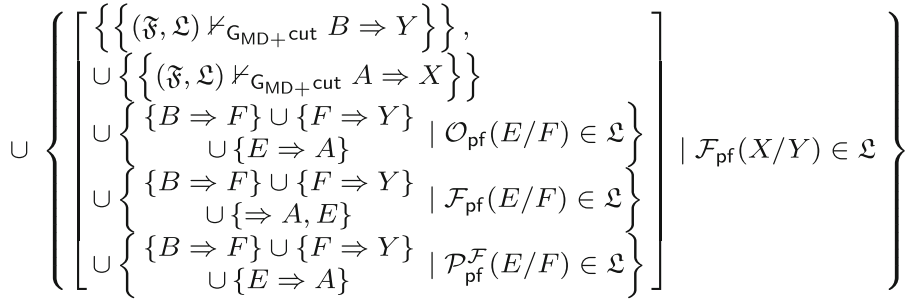

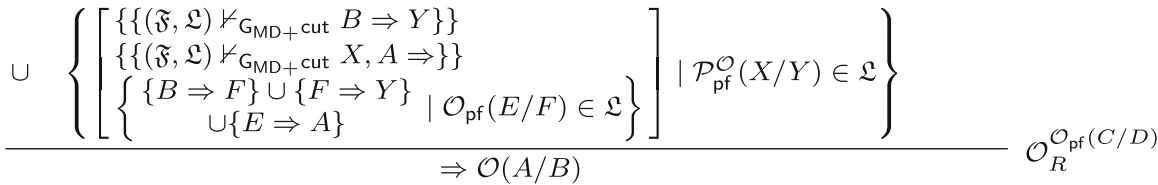

where $\mathcal{O}_{\mathrm{pf}}(C / D) \in \mathfrak{L}$.

Fig. 10 The assumption right rule for obligations in presence of prohibitions and permissions

what is obligatory, i.e., $A$ and $C$, cannot be true at the same time. This is equivalent to stating that we can derive $\neg(A \wedge C)$, or equivalently the sequent $A, C \Rightarrow$ from the facts. In contrast, an obligation $\mathcal{O}_{\mathrm{pf}}(A / B)$ conflicts with a prohibition $\mathcal{F}_{\text {pf }}(C / D)$ if following the obligation would necessarily violate the prohibition, or in other words if the implication $A \rightarrow C$ follows from the facts, i.e., the sequent $A \Rightarrow C$ is derivable from the facts. Two prohibitions $\mathcal{F}_{\mathrm{pf}}(A / B)$ and $\mathcal{F}_{\mathrm{pf}}(C / D)$ then conflict if it is not possible to follow both. This means that the formula $A \vee C$ resp. the sequent $\Rightarrow A, C$ follows from the facts. Finally, a prohibition-permission $\mathcal{P}_{\mathrm{pf}}^{\mathcal{F}}(A / B)$ conflicts with a prohibition $\mathcal{F}_{\mathrm{pf}}(C / D)$ if the permitted $A$ implies the forbidden $C$, i.e., if $A \rightarrow C$ resp. the sequent $A \Rightarrow C$ is derivable.

Incorporating this rationale into the construction of the assumption right rule for obligations leads to the rules $\mathcal{O}_{R}^{\mathcal{O}_{\mathrm{pf}}(C / D)}$ for every formula $\mathcal{O}_{\mathrm{pf}}(C / D) \in \mathfrak{L}$ shown in Fig. 10. 
Similarly, using the above rationale to construct the assumption right rule for prohibitions yields the rule $\mathcal{F}_{R}^{\mathcal{F}_{\text {pf }}(C / D)}$ given in Fig. 11 .

Left rules In order to obtain a cut-free system again we need to absorb cuts between the principal formulae of these two rules and the remaining rules into the rule set. In particular, saturating the rule set under cuts between the assumption right rule for obligations and the rule $D_{\mathcal{O}}$ and the interaction rule $D_{\mathcal{O F}}$ respectively yields the rules $\mathcal{O}_{L}^{\mathcal{O}_{\text {pf }}(C / D)}$ and $\mathcal{F}_{L}^{\mathcal{O}_{\text {pf }}(C / D)}$ shown in Figs. 12 and 13. Using these rules it is possible to derive an obligation and prohibition respectively on the left, from a prima-facie obligation $\mathcal{O}_{\mathrm{pf}}(C / D) \in \mathfrak{L}$. Similarly, cuts between the assumption right rule for prohibitions and the rule $D_{\mathcal{F}}$ and the interaction rule $D_{\mathcal{O} \mathcal{F}}$ respectively yield the rules $\mathcal{F}_{L}^{\mathcal{F}_{\text {pf }}(C / D)}$ and $\mathcal{O}_{L}^{\mathcal{F}_{\text {pf }}(C / D)}$ shown in Figs. 14 and 15 , respectively. Note that in the case where $\mathfrak{L}$ contains only prima-facie obligation formulae we obtain exactly the rules of $\mathrm{ga}_{\mathfrak{L}}$ in Fig. 5 of the previous section.

Again, for each of these rules we divide the premisses into the standard block consisting of the first two premisses, the not-excepted block, consisting of the underivability statements stating that there is no conflicting and at least as specific prima facie deontic statement, and the no-active-conflict block, consisting of the last three sets of premisses and stating that every conflicting prima-facie deontic statement is overruled by a more specific one. For every prima-facie deontic statement, the corresponding possible premisses in the no-active-conflict block again are divided into the no-conflict block consisting of the underivability premisses, and the override block, consisting of the premisses stating that the deontic statement is overridden.

Similarly to the simplified case without prohibitions and permissions, we use the following notation.

Definition 3 We write $(\mathfrak{F}, \mathfrak{L}) \vdash_{\mathrm{G}_{\mathrm{MD}+} \text { cut }} \Gamma \Rightarrow \Delta$ if there is a valid proto-derivation of $\Gamma \Rightarrow \Delta$ from $\mathfrak{F}$ in the system $\mathrm{G}_{\mathrm{MD}+}$ extended with the following global assumption rules for $\mathfrak{L}$ :

$$
\mathrm{ga}_{\mathfrak{L}}:=\left\{\begin{array}{lc}
\text { op } 1_{s}^{\mathrm{op} 2(C / D)}, & \text { op1 } \in\{\mathcal{O}, \mathcal{F}\}, \text { op } 2 \in\left\{\mathcal{O}_{\mathrm{pf}}, \mathcal{F}_{\mathrm{pf}}\right\} \\
\text { op2 }(C / D) \in \mathfrak{L}, s \in\{L, R\}
\end{array}\right\} .
$$

The following lemma shows that the rules $\mathcal{O}_{L}^{\mathcal{O}_{\mathrm{pf}}(C / D)}, \mathcal{F}_{L}^{\mathcal{O}_{\mathrm{pf}}(C / D)}, \mathcal{F}_{L}^{\mathcal{F}_{\mathrm{pf}}(C / D)}$ and $\mathcal{O}_{L}^{\mathcal{F}_{\text {pf }}(C / D)}$ in the presence of the cut rule can be seen as a mere technical convenience, because they do not change the set of derivable sequents. However, in order to be able to perform automated reasoning in our system, we also would like to eliminate the cut rule itself, and the resulting system would not be complete without these rules.

Lemma 1 (Redundancy of the left rules) If there is a valid proto-derivation of $\Gamma \Rightarrow \Delta$ in $G_{M D+}$ cut from $(\mathfrak{F}, \mathfrak{L})$, then there is a valid proto-derivation of $\Gamma \Rightarrow \Delta$ from $(\mathfrak{F}, \mathfrak{L})$ in the system without the rules in Figs. 12, 13, 14 and 15.

Proof We show how to replace every application of one of these rules by cuts and an application of $\mathcal{O}_{R}^{\mathcal{O}_{\text {pf }}(C / D)}$ and $\mathcal{F}_{R}^{\mathcal{F}_{\text {pf }}(C / D)}$ respectively. So consider first an application of the rule $\mathcal{O}_{L}^{\mathcal{O}_{\mathrm{pf}}(C / D)}$ as in Fig. 12. From every premiss of the form $\Gamma, A \Rightarrow \Delta$ and 


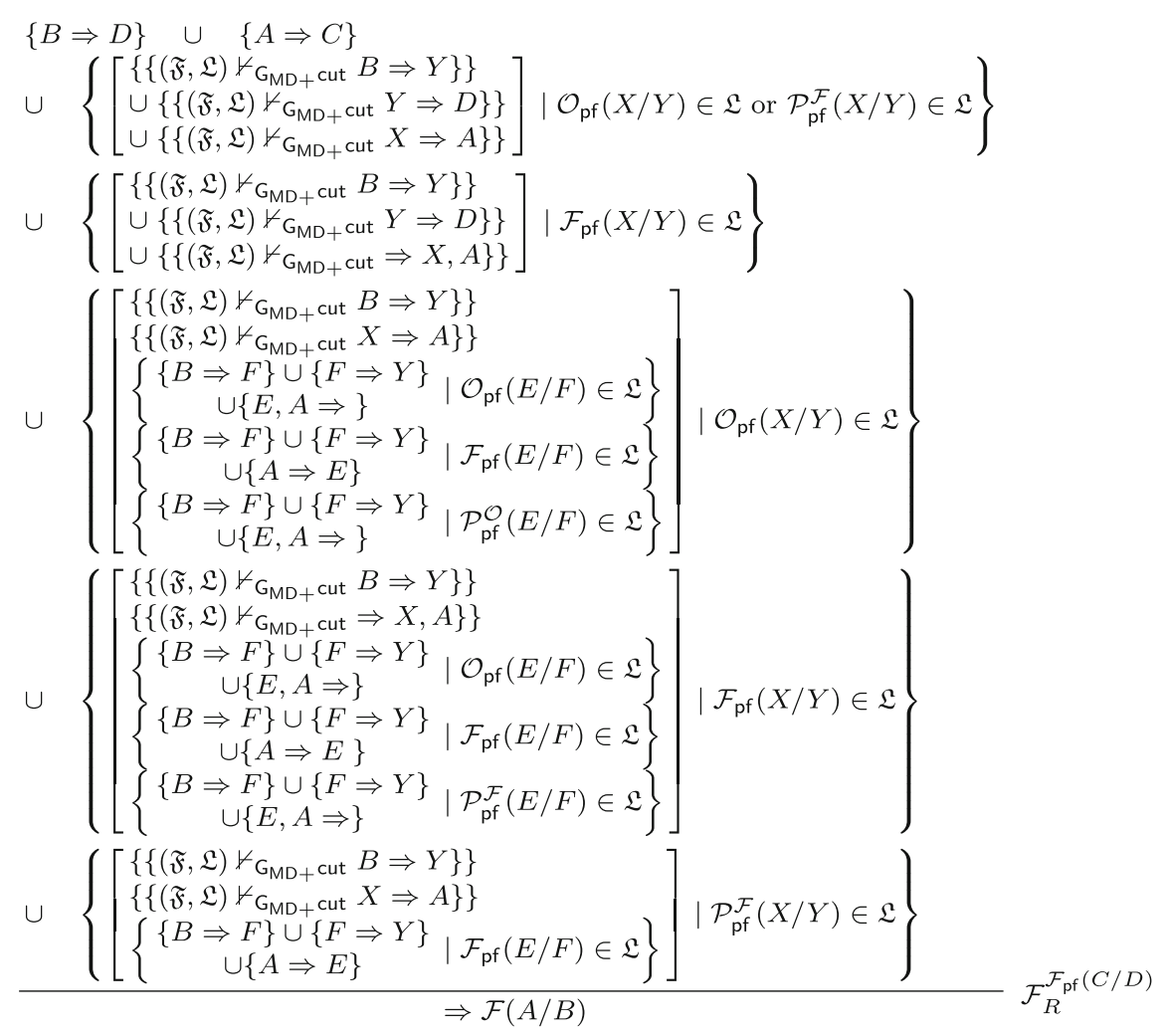

where $\mathcal{F}_{\text {pf }}(C / D) \in \mathfrak{L}$

Fig. 11 The assumption right rule for prohibitions

$\Sigma \Rightarrow A, \Pi$ using weakening and the implication rules we obtain the corresponding premiss $\Gamma \Rightarrow A \rightarrow \perp, \Delta$ and $\Sigma, A \rightarrow \perp \Rightarrow \Pi$, respectively. Further, from every underivability statement $(\mathfrak{F}, \mathfrak{L}) \nvdash_{\mathrm{G}_{\mathrm{MD}+} \text { cut }} \Gamma \Rightarrow A, \Delta$ we obtain the corresponding statement $(\mathfrak{F}, \mathfrak{L}) \nvdash_{\mathrm{G}_{\mathrm{MD}+} \text { cut }} \Gamma, A \rightarrow \perp \Rightarrow \Delta$, since if for the latter there were a valid proto-derivation, we could extend it to one of the former via:

$$
\frac{\frac{\overline{\Gamma, A \Rightarrow A, \perp, \Delta}}{\Gamma \Rightarrow A, A \rightarrow \perp, \Delta} \rightarrow_{R} \quad \Gamma, A \Rightarrow \Delta}{\Gamma \Rightarrow A, \Delta} \text { cut }
$$

Analogously, from every underivability statement $(\mathfrak{F}, \mathfrak{L}) \nvdash_{\mathrm{G}_{\mathrm{MD}+} \mathrm{cut}} \Sigma, A \Rightarrow \Pi$ we obtain the corresponding statement $(\mathfrak{F}, \mathfrak{L}) \nvdash_{\mathrm{G}_{\mathrm{MD}+} \text { cut }} \Sigma \Rightarrow A \rightarrow \perp, \Pi$. Hence we have all the premisses necessary to apply the rule $\mathcal{O}_{R}^{\mathcal{O}_{\mathrm{pf}}(C / D)}$ with conclusion $\Rightarrow$ $\mathcal{O}(A \rightarrow \perp / B)$. From this we obtain the conclusion of the original application of the 


$$
\begin{aligned}
& \{B \Rightarrow D\} \quad \cup \quad\{C, A \Rightarrow\}
\end{aligned}
$$

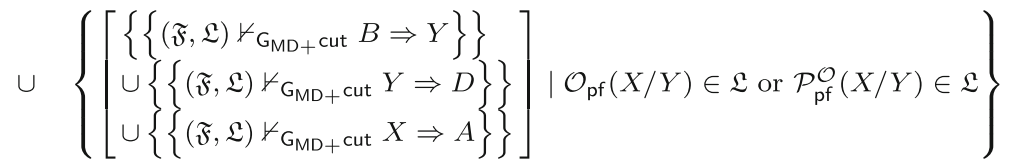

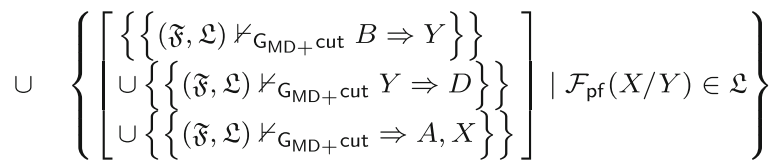

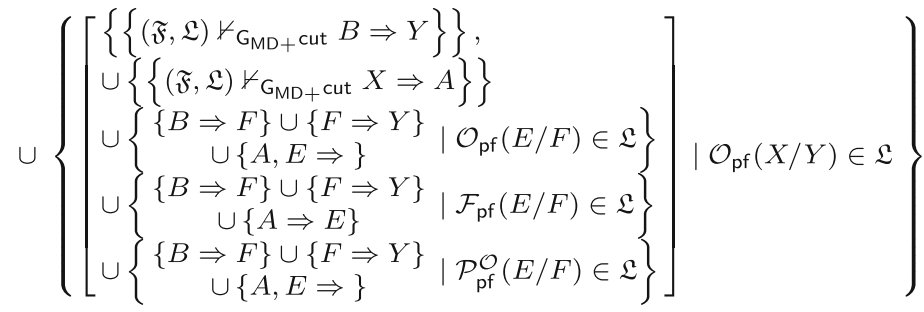

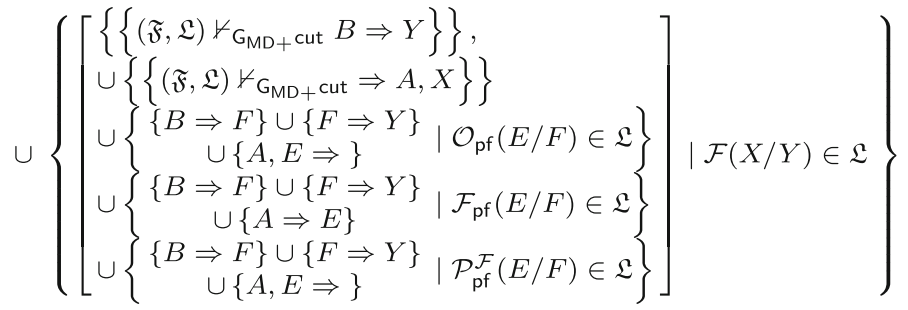

$$
\begin{aligned}
& \frac{\cup\left\{\left[\begin{array}{l}
\left\{\left\{(\mathfrak{F}, \mathfrak{L}) \nvdash \mathrm{G}_{\mathrm{MD}+\text { cut }} B \Rightarrow Y\right\}\right\} \\
\left\{\left\{(\mathfrak{F}, \mathfrak{L}) \nvdash \mathrm{G}_{\mathrm{MD}+\text { cut }} X \Rightarrow A\right\}\right\} \\
\left.\left\{\begin{array}{c}
\{B \Rightarrow F\} \cup\{F \Rightarrow Y\} \\
\cup\{E, A \Rightarrow\}
\end{array}\right] \mid \mathcal{O}_{\mathrm{pf}}(E / F) \in \mathfrak{L}\right\}
\end{array}\right] \mathcal{P}_{\mathrm{pf}}^{\mathcal{O}(X / Y) \in \mathfrak{L}\}}\right.}{\mathcal{O}(A / B) \Rightarrow} \mathcal{O}_{L}^{\mathcal{O}_{\mathrm{pf}}(C / D)}
\end{aligned}
$$

where $\mathcal{O}_{\mathrm{pf}}(C / D) \in \mathfrak{L}$.

Fig. 12 The assumption left rule for obligations in presence of prohibitions and permissions

rule $\mathcal{O}_{L}^{\mathcal{O}_{\text {pf }}(C / D)}$ using cut on the conclusion of the rule $\mathrm{D}_{\mathcal{O}}$ as follows:

$$
\frac{\Rightarrow \mathcal{O}(A \rightarrow \perp / B) \frac{A \rightarrow \perp, A \Rightarrow B \Rightarrow B}{\mathcal{O}(A / B), \mathcal{O}(A \rightarrow \perp / B) \Rightarrow}}{\mathcal{O}(A / B) \Rightarrow} \mathrm{D}_{\mathcal{O}}
$$

The premisses are clearly derivable.

In a similar way we obtain the conclusion of an application of $\mathcal{F}_{L}^{\mathcal{O}_{\mathrm{pf}}(C / D)}$ from an application of $\mathcal{O}_{R}^{\mathcal{O}_{\text {pf }}(C / D)}$ and a cut with the conclusion of the rule $\mathrm{D}_{\mathcal{O F}}$. The reasoning for the remaining rules is analogous.

The central technical result about the system stating elimination of the cut rule then follows a reasonably standard pattern of a cut elimination proof, but slightly adjusted 


$$
\begin{aligned}
& \{B \Rightarrow D\} \quad \cup \quad\{C \Rightarrow A\}
\end{aligned}
$$

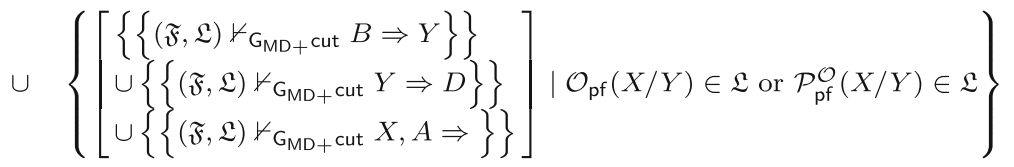

$$
\begin{aligned}
& \cup\left\{\left[\begin{array}{c}
\left.\left\{\begin{array}{l}
\left\{(\mathfrak{F}, \mathfrak{L}) \nvdash_{\mathrm{G}_{\mathrm{MD}+\text { cut }}} B \Rightarrow Y\right\} \\
\cup\left\{\left\{(\mathfrak{F}, \mathfrak{L}) \nvdash_{\mathrm{G}_{\mathrm{MD}+} \text { cut }} Y \Rightarrow D\right.\right. \\
\cup\left\{\left\{(\mathfrak{F}, \mathfrak{L}) \nvdash_{\mathrm{G}_{\mathrm{MD}+\text { cut }}} A \Rightarrow X\right.\right.
\end{array}\right\}\right\}
\end{array}\right\} \mid \mathcal{F}_{\mathrm{pf}}(X / Y) \in \mathfrak{L}\right\}
\end{aligned}
$$

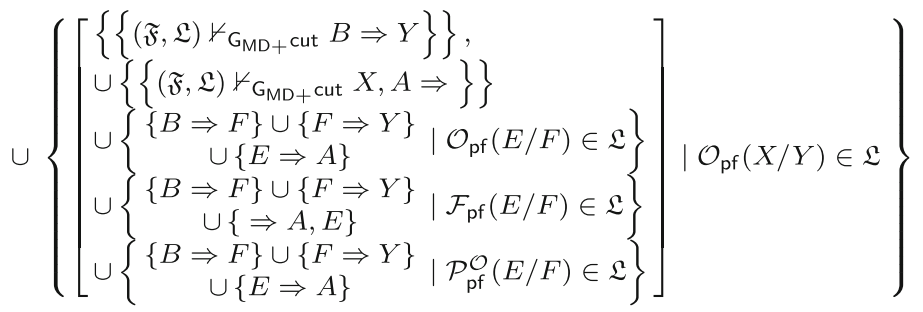

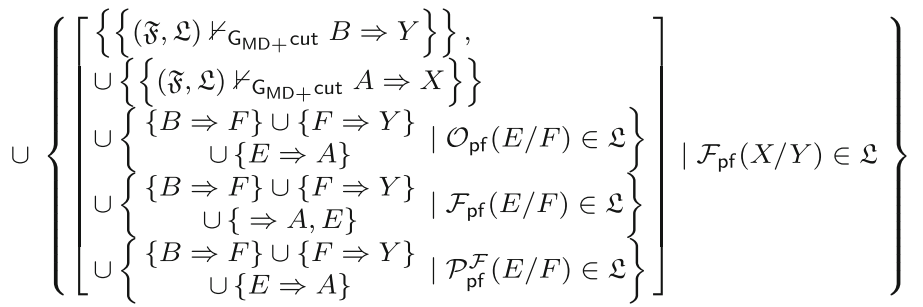

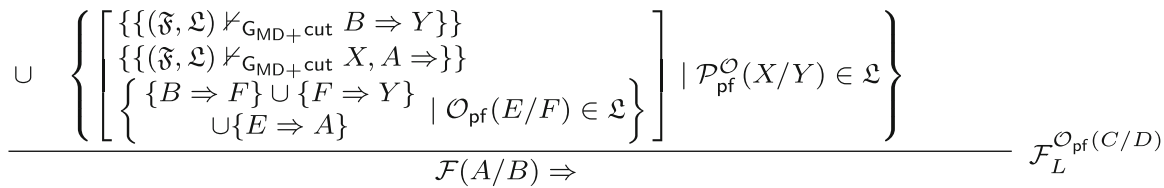

where $\mathcal{O}_{\mathrm{pf}}(C / D) \in \mathfrak{L}$.

Fig. 13 The derived left rule for prohibitions in presence of prohibitions and permissions

to also accommodate for the underivability statements. The proof is detailed in the "Appendix". In the following we write $(\mathfrak{F}, \mathfrak{L}) \vdash_{\mathrm{G}_{\mathrm{MD}+}}$ for the cut-free system, i.e., the calculus $(\mathfrak{F}, \mathfrak{L}) \vdash_{\mathrm{G}_{\mathrm{MD}+} \text { cut }}$ with the cut rule removed. Note that again in the cut-free calculus $\mathrm{G}_{\mathrm{MD}+}$ the non-derivability statements range over the system with the cut rule.

Theorem 2 (Cut elimination) If (F, $\mathfrak{L}) \vdash_{G_{M D+} \text { cut }} \Gamma \Rightarrow \Delta$, then $(\mathfrak{F}, \mathfrak{L}) \vdash_{G_{M D+}} \Gamma \Rightarrow \Delta$. Proof See the "Appendix".

From the cut elimination theorem we then obtain equivalence of the systems with and without the cut rule:

Proposition 3 For every $\mathfrak{F}$, $\mathfrak{L}$ we have

$$
(\mathfrak{F}, \mathfrak{L}) \vdash_{G_{M D+} \text { cut }} \Gamma \Rightarrow \Delta \text { if and only if }(\mathfrak{F}, \mathfrak{L}) \vdash_{G_{M D+}} \Gamma \Rightarrow \Delta \text {. }
$$




$$
\begin{aligned}
& \{B \Rightarrow D\} \quad \cup \quad\{\Rightarrow A, C\} \\
& \cup\left\{\left[\begin{array}{l}
\left\{\left\{(\mathfrak{F}, \mathfrak{L}) \nvdash_{\mathrm{G}_{\mathrm{MD}+} \text { cut }} B \Rightarrow Y\right\}\right\} \\
\cup\left\{\left\{(\mathfrak{F}, \mathfrak{L}) \nvdash_{\mathrm{G}_{\mathrm{MD}+} \text { cut }} Y \Rightarrow D\right\}\right\} \\
\cup\left\{\left\{(\mathfrak{F}, \mathfrak{L}) \nvdash_{\mathrm{G}_{\mathrm{MD}+} \text { cut }} X, A \Rightarrow\right\}\right\}
\end{array}\right] \mid \mathcal{O}_{\mathrm{pf}}(X / Y) \in \mathfrak{L} \text { or } \mathcal{P}_{\mathrm{pf}}^{\mathcal{F}}(X / Y) \in \mathfrak{L}\right\} \\
& \cup\left\{\left[\begin{array}{l}
\left\{\left\{(\mathfrak{F}, \mathfrak{L}) \nvdash_{\mathrm{G}_{\mathrm{MD}+} \text { cut }} B \Rightarrow Y\right\}\right\} \\
\cup\left\{\left\{(\mathfrak{F}, \mathfrak{L}) \nvdash_{\mathrm{G}_{\mathrm{MD}+} \text { cut }} Y \Rightarrow D\right\}\right\} \\
\cup\left\{\left\{(\mathfrak{F}, \mathfrak{L}) \nvdash_{\mathrm{G}_{\mathrm{MD}+\text { cut }}} A \Rightarrow X\right\}\right\}
\end{array}\right] \mid \mathcal{F}_{\mathrm{pf}}(X / Y) \in \mathfrak{L}\right\}
\end{aligned}
$$

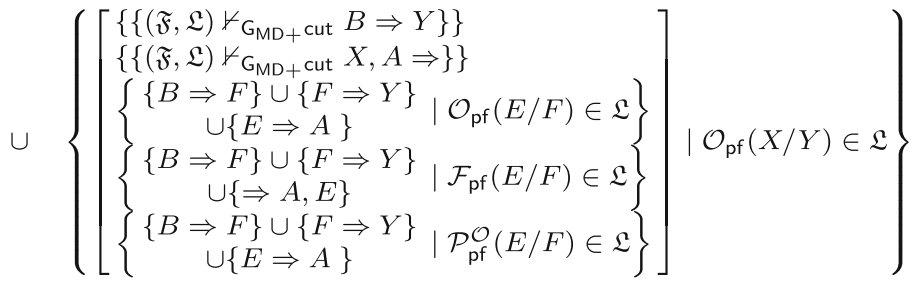

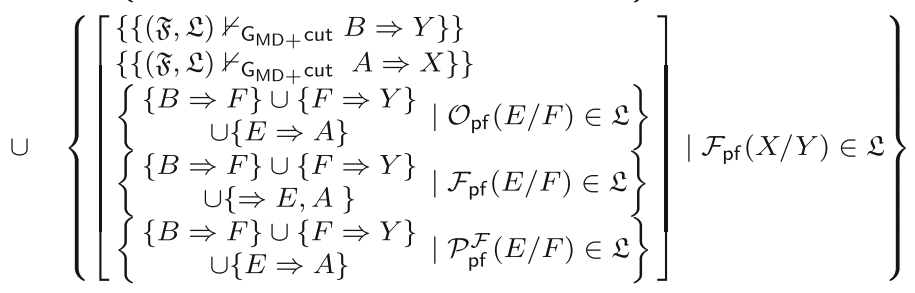

$$
\begin{aligned}
& \cup\left\{\begin{array}{c}
\left.\left[\begin{array}{l}
\left\{\left\{(\mathfrak{F}, \mathfrak{L}) \nvdash \mathrm{G}_{\mathrm{MD}+\text { cut }} B \Rightarrow Y\right\}\right\} \\
\left\{\left\{(\mathfrak{F}, \mathfrak{L}) \nvdash \mathrm{G}_{\mathrm{MD}+\text { cut }} X, A \Rightarrow\right\}\right\} \\
\left.\left\{\begin{array}{c}
\{B \Rightarrow F\} \cup\{F \Rightarrow Y\} \\
\cup\{\Rightarrow A, E\}
\end{array}\right] \mid \mathcal{F}_{\mathrm{pf}}(E / F) \in \mathfrak{L}\right\}
\end{array}\right] \mid \mathcal{P}_{\mathrm{pf}}^{\mathcal{F}(X / Y) \in \mathfrak{L}}\right\} \\
\mathcal{F}(A / B) \Rightarrow
\end{array} \mathcal{F}_{L}^{\mathcal{F}_{\mathrm{pf}}(C / D)}\right.
\end{aligned}
$$

where $\mathcal{F}_{\text {pf }}(C / D) \in \mathfrak{L}$

Fig. 14 The assumption left rule for prohibitions

Proof The "only if" direction is the statement of the cut elimination theorem. The proof for the "if" direction is straightforward, since every rule in $\mathrm{G}_{M D}$ is also a rule in $\mathrm{G}_{\mathrm{MD}}$ cut, and since the underivability statements range over the same system for valid proto-derivations in both systems.

\subsection{Consequences of cut elimination}

The Cut Elimination Theorem has a number of important consequences, in particular the fact that the notion of a valid proto-derivation is well-defined, consistency of the system, and a decidability and complexity result. The latter shows that despite the somewhat complicated shape of the assumption rules, reasoning in the calculus does not have a higher complexity than reasoning in standard modal logics such as $\mathrm{K}$ or in intuitionistic logic.

The fact that valid proto-derivations are well-defined can be seen by considering the following alternative stratified definition. 


$$
\begin{aligned}
& \{B \Rightarrow D\} \quad \cup \quad\{A \Rightarrow C\} \\
& \cup\left\{\left[\begin{array}{l}
\left\{\left\{(\mathfrak{F}, \mathfrak{L}) \nvdash_{\mathrm{G}_{\mathrm{MD}+} \text { cut }} B \Rightarrow Y\right\}\right\} \\
\cup\left\{\left\{(\mathfrak{F}, \mathfrak{L}) \nvdash_{\mathrm{G}_{\mathrm{MD}+} \text { cut }} Y \Rightarrow D\right\}\right\} \\
\cup\left\{\left\{(\mathfrak{F}, \mathfrak{L}) \nvdash_{\mathrm{G}_{\mathrm{MD}+} \text { cut }} X \Rightarrow A\right\}\right\}
\end{array}\right] \mid \mathcal{O}_{\mathrm{pf}}(X / Y) \in \mathfrak{L} \text { or } \mathcal{P}_{\mathrm{pf}}^{\mathcal{F}}(X / Y) \in \mathfrak{L}\right\} \\
& \cup\left\{\left[\begin{array}{l}
\left\{\left\{(\mathfrak{F}, \mathfrak{L}) \nvdash_{\mathrm{G}_{\mathrm{MD}+} \text { cut }} B \Rightarrow Y\right\}\right\} \\
\cup\left\{\left\{(\mathfrak{F}, \mathfrak{L}) \nvdash_{\mathrm{G}_{\mathrm{MD}+} \text { cut }} Y \Rightarrow D\right\}\right\} \\
\cup\left\{\left\{(\mathfrak{F}, \mathfrak{L}) \nvdash_{\mathrm{G}_{\mathrm{MD}+} \text { cut }} \Rightarrow X, A\right\}\right\}
\end{array}\right] \mid \mathcal{F}_{\mathrm{pf}}(X / Y) \in \mathfrak{L}\right\}
\end{aligned}
$$

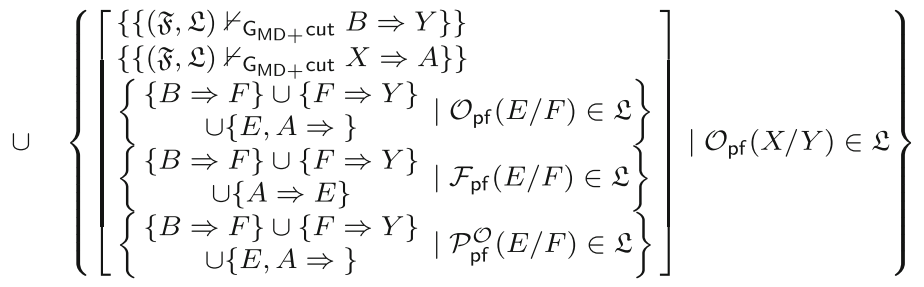

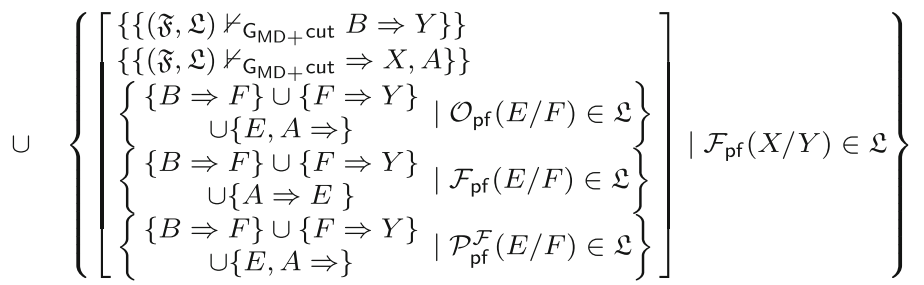

$$
\begin{aligned}
& \cup \frac{\left\{\left[\begin{array}{l}
\left\{\left\{(\mathfrak{F}, \mathfrak{L}) \nvdash \mathrm{G}_{\mathrm{MD}+\text { cut }} B \Rightarrow Y\right\}\right\} \\
\left\{\left\{(\mathfrak{F}, \mathfrak{L}) \nvdash \mathrm{G}_{\mathrm{MD}+\text { cut }} X \Rightarrow A\right\}\right\} \\
\left.\left\{\begin{array}{c}
\{B \Rightarrow F\} \cup\{F \Rightarrow Y\} \\
\cup\{A \Rightarrow E\}
\end{array}\right] \mid \mathcal{F}_{\mathrm{pf}}(E / F) \in \mathfrak{L}\right\}
\end{array}\right] \mid \mathcal{P}_{\mathrm{pf}}^{\mathcal{F}(X / Y) \in \mathfrak{L}}\right\}}{\mathcal{O}(A / B) \Rightarrow} \mathcal{O}_{L}^{\mathcal{F}_{\text {pf }}(C / D)}
\end{aligned}
$$

where $\mathcal{F}_{\text {pf }}(C / D) \in \mathfrak{L}$

Fig. 15 The new assumption left rule for obligations

Definition 4 A proto-derivation of rank $n$ in $\mathrm{G}_{\mathrm{MD}+}$ (in $\mathrm{G}_{\mathrm{MD}+} \mathrm{cut}$ ) from $(\mathfrak{F}, \mathfrak{L})$ with conclusion $\Gamma \Rightarrow \Delta$ is a proto-derivation in $\mathrm{G}_{\mathrm{MD}+}$ (in $\mathrm{G}_{\mathrm{MD}+}$ cut) from $(\mathfrak{F}, \mathfrak{L})$ with conclusion $\Gamma \Rightarrow \Delta$ such that

- every formula occurring in the proto-derivation has modal nesting depth at most $n$

- every formula occurring in an underivability statement in the proto-derivation has modal nesting depth at most $n-1$.

If $n$ is a natural number, then a proto-derivation is $n$-valid if it is of rank $n$ and for every $k<n$, for none of the underivability statements occurring in it there is a $k$-valid proto-derivation in $\mathrm{G}_{\mathrm{MD}+}$ cut from $(\mathfrak{F}, \mathfrak{L})$.

Since the modal nesting depth of the formulae in the underivability statements in the rules of $\mathrm{ga}_{\mathfrak{L}}$ is stricly lower than that of the formulae in the conclusion, the question whether a proto-derivation is $n$-valid only depends on $k$-validity for $k<n$. Hence this definition is inductive and not circular. Using the Cut Elimination Theorem we obtain that it is equivalent to unrestricted validity of proto-derivations as follows. 
Theorem 3 For every sequent $\Gamma \Rightarrow \Delta$ with modal nesting depth at most $n$ there is a valid proto-derivation in $G_{M D+}$ cut from $(\mathfrak{F}, \mathfrak{L})$ with conclusion $\Gamma \Rightarrow \Delta$ if and only if there is a n-valid proto-derivation in $G_{M D+}$ cut from $(\mathfrak{F}, \mathfrak{L})$ with conclusion $\Gamma \Rightarrow \Delta$.

Proof By induction on $n$.

So suppose the statement holds for every $k<n$. If there is a valid proto-derivation of $\Gamma \Rightarrow \Delta$ from $(\mathfrak{F}, \mathfrak{L})$ in $\mathrm{G}_{\mathrm{MD}+}$ cut with conclusion $\Gamma \Rightarrow \Delta$, then by Cut Elimination (Theorem 2) there is a valid proto-derivation of $\Gamma \Rightarrow \Delta$ from $(\mathfrak{F}, \mathfrak{L})$ in $\mathrm{G}_{\mathrm{MD}+}$. Since none of the rules of $\mathrm{G}_{\mathrm{MD}+}$ or $\mathrm{ga}_{\mathfrak{L}}$ increases the modal nesting depth from conclusion to premiss(es), the maximal modal nesting depth of formulae occurring in this proto-derivation is $n$. Further, since the modal nesting depth of the formulae in the underivability statements in the rules $\mathrm{ga}_{\mathfrak{L}}$ is strictly smaller than that of the conclusion, every formula occurring in an underivability statement in this proto-derivation has modal nesting depth at most $n-1$. Hence the proto-derivation is of rank $n$. Since the modal nesting depth of the formulae in the underivability statements is at most $n-1$, by induction hypothesis we obtain that for these there is no $k$ valid proto-derivation for any $k \leq n-1$.

Hence the proto-derivation is $n$-valid. Conversely, if we have a $n$-valid protoderivation, then again by induction hypothesis we obtain that for none of the underivability statements occurring in it there is a valid proto-derivation. Since a proto-derivation of rank $n$ in particular is a proto-derivation, we obtain a valid protoderivation for the same conclusion.

Well-definedness of the notion of a valid proto-derivation then follows immediately from the previous theorem together with the fact that $n$-validity is well-defined:

Corollary 1 (Well-definedness) The notion of a valid proto-derivation is well-defined.

As a second consequence of Cut Elimination we obtain that the rules ga $\mathfrak{L}_{\mathfrak{L}}$ are compatible with the logic MD+ as given in Figs. 1 and 7 in the sense that they do not yield any conflicting obligations or prohibitions:

Theorem 4 (Consistency) For any $\mathfrak{L}$ and $\mathfrak{F}$ not containing the empty sequent, the consequences of $\mathfrak{L}$ under $\mathfrak{F}$ are consistent over $M D+$, i.e., $(\mathfrak{F}, \mathfrak{L}) \nvdash_{G_{M D+} \text { cut }} \Rightarrow \perp$. Hence in particular

- there are no $A, B$ with $(\mathfrak{F}, \mathfrak{L}) \vdash_{G_{M D+} \text { cut }} \Rightarrow \mathcal{O}(A / B) \wedge \mathcal{O}(\neg A / B)$;

- there are no $A, B$ with $(\mathfrak{F}, \mathfrak{L}) \vdash_{G_{M D+} \text { cut }} \Rightarrow \mathcal{F}(A / B) \wedge \mathcal{F}(\neg A / B)$;

- there are no $A, B$ with $(\mathfrak{F}, \mathfrak{L}) \vdash_{G_{M D+}}$ cut $\Rightarrow \mathcal{O}(A / B) \wedge \mathcal{F}(A / B)$;

- there is no $B$ with $(\mathfrak{F}, \mathfrak{L}) \vdash_{G_{M+}+\text { cut }} \Rightarrow \mathcal{R}(\perp / B)$.

Proof By inspection it is clear that all the rules in the calculus $\mathrm{G}_{\mathrm{MD}+\mathrm{ga}} \mathfrak{L}$ have the $s u b$ formula property relative to $\mathfrak{L}$ in the sense that every formula occurring in a premise of a rule, including the underivability statements, is a subformula of a formula occurring in its conclusion or in $\mathfrak{L}$. Since the empty sequent is not in $\mathfrak{F}$, and apart from $\mathrm{W}_{R}$ there is no rule introducing $\perp$ on the right hand side of a sequent, we cannot derive $\Rightarrow \perp$. The second statement follows from this using derivability of $\mathcal{O}(A / B) \wedge \mathcal{O}(\neg A / B) \Rightarrow$ and the analogous sequents for the statements involving $\mathcal{F}$ and $\mathcal{R}$ together with the cut rule. 


$$
\begin{aligned}
& \overline{\Gamma, p \Rightarrow p, \Delta} \text { init } \frac{\Gamma, B \Rightarrow \Delta \quad \Gamma \Rightarrow A, \Delta}{\Gamma, \perp \Rightarrow \Delta} \perp_{L} \quad \frac{\Gamma, A \Rightarrow B, \Delta}{\Gamma, A \rightarrow B \Rightarrow \Delta} \rightarrow_{L} \\
& \frac{A \Rightarrow C \quad B \Rightarrow D \quad D \Rightarrow B}{\Gamma, \mathcal{O}(A / B) \Rightarrow \mathcal{O}(C / D), \Delta} \operatorname{Mon}_{\mathcal{O}} \quad \frac{A, C \Rightarrow \quad B \Rightarrow D \quad D \Rightarrow B}{\Gamma, \mathcal{O}(A / B), \mathcal{O}(C / D) \Rightarrow \Delta} \mathrm{D}_{\mathcal{O}} \frac{A \Rightarrow}{\Gamma, \mathcal{O}(A / B) \Rightarrow \Delta} \mathrm{P}_{\mathcal{O}} \\
& \frac{C \Rightarrow A \quad B \Rightarrow D \quad D \Rightarrow B}{\Gamma, \mathcal{F}(A / B) \Rightarrow \mathcal{F}(C / D), \Delta} \operatorname{Mon}_{\mathcal{F}} \quad \frac{\Rightarrow A, B \quad C \Rightarrow D \quad D \Rightarrow C}{\Gamma, \mathcal{F}(A / C), \mathcal{F}(B / D) \Rightarrow \Delta} \mathrm{D}_{\mathcal{F}} \frac{\Rightarrow A}{\Gamma, \mathcal{F}(A / B) \Rightarrow \Delta} \mathrm{P}_{\mathcal{F}} \\
& \frac{A \Rightarrow C \quad B \Rightarrow D \quad D \Rightarrow B}{\Gamma, \mathcal{R}(A / B) \Rightarrow \mathcal{R}(C / D), \Delta} \operatorname{Mon}_{\mathcal{R}} \frac{A \Rightarrow}{\Gamma, \mathcal{R}(A / B) \Rightarrow \Delta} \mathrm{P}_{\mathcal{R}} \frac{A \Rightarrow C \quad B \Rightarrow D \quad D \Rightarrow B}{\Delta, \mathcal{O}(A / B), \mathcal{F}(C / D) \Rightarrow \Delta} \mathrm{D}_{\mathcal{O F}}
\end{aligned}
$$

Fig. 16 The system $\mathrm{G} 3_{\mathrm{MD}}$ + without the assumption rules

The third major consequence of the Cut Elimination Theorem is that it permits the restriction of proof search to proto-derivations in the system without the cut rule. Using the (extended) subformula property of the rules of the cut-free system this yields a decision procedure for the logic. To make this precise, the derivability problem is given by the following:

\begin{tabular}{|ll|}
\hline Mīmāmsāa derivability using specificity \\
\hline Input: & $\begin{array}{l}\text { Finite lists } \mathfrak{F}, \mathfrak{L} \text { of propositional facts and prima-facie } \\
\text { deontic statements, and a sequent } \Gamma \Rightarrow \Delta\end{array}$ \\
Question: & Do we have $(\mathfrak{F}, \mathfrak{L}) \vdash_{\mathrm{G}_{\mathrm{MD}+}}$ cut $\Gamma \Rightarrow \Delta ?$ \\
\hline
\end{tabular}

We will show a decidability and complexity result via a natural implementation of backwards proof search on an alternating Turing machine (see Chandra et al. 1981 for details). As usual, for this we first eliminate the structural rules from the system.

Definition 5 The system $G 3_{M D+}$ is the system in Fig. 16, obtained from $G_{M D+}$ by restricting initial sequents to atomic formulae, dropping the weakening and contraction rules, and absorbing weakening into the conclusion of the logical rules. Similarly, for a list $\mathfrak{L}$ of prima-facie deontic statements, the rules $\mathrm{ga}_{\mathfrak{L}}^{*}$ are the rules from $\mathrm{ga}_{\mathfrak{L}}$ with weakening absorbed into the conclusion (only!). E.g., the rule $\mathcal{O}_{R}^{\mathcal{O}_{\text {pf }}(C / D)^{*}}$ has exactly the same premisses as the rule $\mathcal{O}_{R}^{\mathcal{O}_{\text {pf }}(C / D)}$ from Fig. 10, but the conclusion $\Gamma \Rightarrow \mathcal{O}(A / B), \Delta$. A valid proto-derivation in $\mathrm{G} 3_{\mathrm{MD}}+$ from $(\mathfrak{F}, \mathfrak{L})$ is defined as for $\mathrm{G}_{\mathrm{MD}+}$ with the exception that leaves may also be labelled with sequents $\Gamma, \Sigma \Rightarrow$ $\Pi, \Delta$, where $\Sigma \Rightarrow \Pi \in \mathfrak{F}$ and $\Gamma \Rightarrow \Delta$ is an arbitrary sequent. In particular, the underivability statements also range over $\mathrm{G}_{\mathrm{MD}+}$ cut.

The following properties of the calculus are shown by standard methods.

Lemma 2 (Generalised initial sequents) The generalised initial sequent rule

$$
\overline{\Gamma, A \Rightarrow A, \Delta}
$$

is admissible in $\mathrm{G}_{\mathrm{MD}} \mathrm{M}$.

Proof By induction on the complexity of the formula $A$, using the rules $\operatorname{Mon}_{\mathcal{O}}, \operatorname{Mon}_{\mathcal{F}}$, $\operatorname{Mon}_{\mathcal{R}}$ in case the outermost connective is one of $\mathcal{O}, \mathcal{F}, \mathcal{R}$. 
Lemma 3 (Invertibility of the propositional rules) The rules $\rightarrow_{R}^{*}$ and $\rightarrow{ }_{L}^{*}$ are depthpreserving invertible in $\mathrm{G}_{\mathrm{MD}+}$, i.e., whenever there is a valid proto-derivation of their conclusion in $G 3_{M D+}$ with depth $n$ from $(\mathfrak{F}, \mathfrak{L})$, then for each of the premisses there is a valid proto-derivation with depth $n$ from $(\mathfrak{F}, \mathfrak{L})$ as well.

Proof By induction on the depth of the valid proto-derivation.

Lemma 4 (Admissibility of weakening and contraction) The weakening and the contraction rules are depth-preserving admissible, i.e., whenever there are valid protoderivations in $G 3_{M D}$ of the premisses of these rules with depth $n$ from $(\mathfrak{F}, \mathfrak{L})$, then there are valid proto-derivations of their conclusions with the same depth from $(\mathfrak{F}, \mathfrak{L})$ as well.

Proof By induction on the depth of the valid proto-derivation, using Lemma 3 in case the contracted formula is a principal formula in a propositional rule.

Lemma 5 Let $\mathfrak{F}, \mathfrak{L}$ be finite lists of propositional facts and prima-facie deontic statements, respectively, and let $\Gamma \Rightarrow \Delta$ be a sequent. Then $(\mathfrak{F}, \mathfrak{L}) \vdash_{G_{M D+}} \Gamma \Rightarrow \Delta$ if and only if $(\mathfrak{F}, \mathfrak{L}) \vdash_{G 3_{M D+}} \Gamma \Rightarrow \Delta$.

Proof Since the underivability statement range over the same system, we only need to show how to convert proto-derivations from one system to the other. For the "only if" direction, we replace applications of the weakening and contraction rules with invocations of Lemma 4, and simulate the generalised intitial sequents of $\mathrm{G}_{\mathrm{MD}}+\mathrm{using}$ Lemma 2. For the "if" direction, we make the absorbed weakening explicit using the rules $\mathrm{W}_{L}, \mathrm{~W}_{R}$.

Using the previous lemma, to solve the Mīmāmsā derivability problem, it is then enough to perform backwards proof search in the system $G 3_{M D+}$ with the rules ga ${ }_{\mathfrak{L}}^{*}$. Recall that for the assumption rules from Figs. 10, 11, 12, 13, 14 and 15 we divide the schematic premisses into blocks: the standard block contains the first two premisses; the not-excepted block contains the schematic premisses of the sets in the second and third line, i.e., all those underivability statements stating that the prima-facie deontic statement is not overridden by a more specific one; the no-active-conflict block contains the schematic premisses of the remaining sets. The premisses of the no-active-conflict blocks for each formula from $\mathfrak{L}$ are further divided into the conflict block consisting of the first two premisses in the [.] construct and the override block consisting of the remaining ones (which again depend on additional formulae from $\mathfrak{L}$ ).

Theorem 5 (Decidability and complexity) The Mīmāmsā derivability problem using specificity is decidable in polynomial space.

Proof The implementation of the decision procedure on an alternating Turing machine is shown as Algorithm 1. Intuitively, the algorithm makes existential guesses for the last applied rule, then makes universal choices to verify that every premiss is derivable.

Claim 1 Algorithm 1 terminates in polynomial time.

Suppose that $n$ is the size of the input, i.e., the sum of the number of symbols in $\mathfrak{F}, \mathfrak{L}$ and $\Gamma \Rightarrow \Delta$. Let the complexity of a sequent be the number of occurrences of propositional or modal connectives in it. Every application of a propositional rule removes 


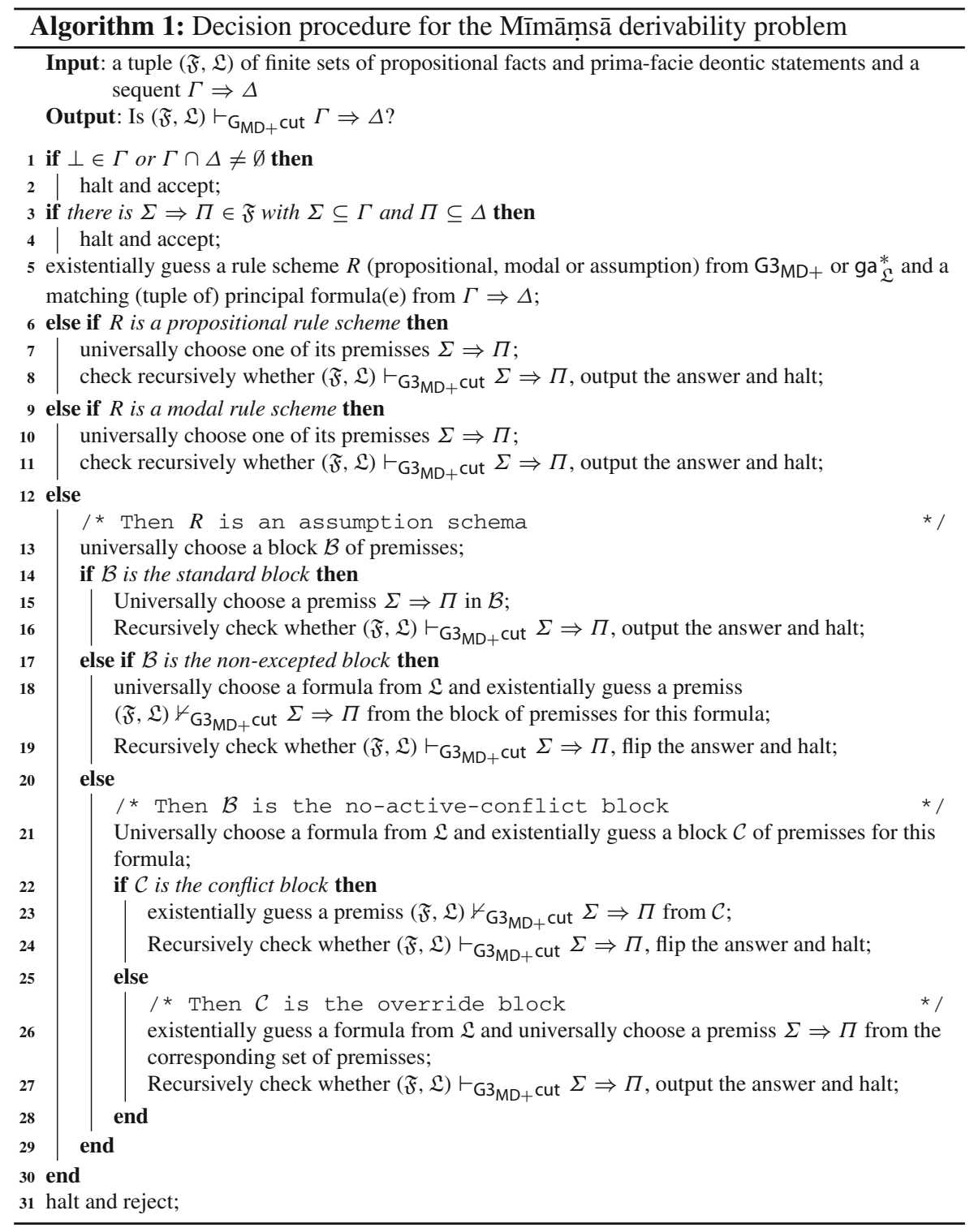

a propositional connective by replacing a propositional formula with its immediate subformulae, and hence reduces the complexity of the sequents. Hence the number of such applications is bounded by the number of subformulae of the conclusion, $\mathfrak{F}$ or $\mathfrak{L}$, and thus bounded by $n$. Moreover, from the shape of the modal and assumption rules together with the fact that the assumptions in $\mathfrak{L}$ do not contain nested modal operators it follows that each of the recursive calls in lines 11, 16, 19, 24 and 27 is on a sequent of strictly lower maximal nesting depth of the modal operators. Hence the 
maximal modal nesting depth of a sequent is bounded by the size $n$ of the input as well, and the maximal number of formulae in a sequent is thus bounded by $2 n$. Due to the fact that there are only finitely many different rule schemes, all the existential and universal choices can be encoded by a suitable combination of a rule scheme, principal formula(e), or sequents of size bounded by $2 n$, consisting only of subformulae of the conclusion. This yields witnesses of size polynomial in $n$ for each of the nondeterministic steps. Moreover, since each recursive call either reduces the complexity or decreases the maximal modal nesting depth, a run of the algorithm makes at most $\mathcal{O}\left(n^{2}\right)$ recursive calls, after which it either accepts with lines 2 or 4 or rejects with line 31. Thus, the algorithm terminates after at most $\mathcal{O}\left(n^{2}\right)$ steps.

Claim 2 Algorithm 1 accepts an input $(\mathfrak{F}, \mathfrak{L}), \Gamma \Rightarrow \Delta$ if and only if $(\mathfrak{F}, \mathfrak{L}) \vdash_{\mathrm{G}_{\mathrm{MD}+} \text { cut }} \Gamma \Rightarrow \Delta$.

We show the claim by induction on the maximal modal nesting depth of $\Gamma \Rightarrow \Delta$. If $(\mathfrak{F}, \mathfrak{L}) \vdash_{\mathrm{G}_{\mathrm{MD}+} \text { cut }} \Gamma \Rightarrow \Delta$, then there is a valid proto-derivation for $\Gamma \Rightarrow \Delta$ in $\mathrm{G}_{\mathrm{MD}+}$ cut. Hence by Cut Elimination (Theorem 2) and Lemma 5 there is a valid protoderivation for $\Gamma \Rightarrow \Delta$ in $\mathrm{G}_{\mathrm{MD}+}$. By induction hypothesis we know that the algorithm rejects all the underivability statements occurring in this proto-derivation. Hence the existential and universal choices corresponding to the rules of the proto-derivation together with recursive calls of the algorithm witness that the algorithm accepts the input. Conversely, from an accepting run of the algorithm we obtain first a cut-free proto-derivation of $\Gamma \Rightarrow \Delta$ in $\mathrm{G}^{\mathrm{MD}}+$ by applying the rules corresponding to the existential choices of the algorithm. Then by induction hypothesis we obtain that none of the underivability statements occurring in this proto-derivation are derivable in $\mathrm{G}_{\mathrm{MD}+} \mathrm{Cut}$ from $(\mathfrak{F}, \mathfrak{L})$, and hence the proto-derivation is valid. Now Lemma 5 yields a valid proto-derivation in $\mathrm{G}_{\mathrm{MD}+}$ and hence in $\mathrm{G}_{\mathrm{MD}+}$ cut.

The two claims together show that Algorithm 1 decides the Mīmāmsāa derivability problem in alternating polynomial time, which is equivalent to polynomial space (Chandra et al. 1981).

A Prolog implementation of the decision procedure is available under http://subsell. logic.at/bprover/deonticProver/version1.2/.

\section{Applications: deciding between different interpretations}

Possible applications of the introduced system are provided by the problem of validating the interpretation or formalisation of a normative text, and in particular the related problem of deciding between different interpretations or formalisations. Both of these problems are of course common to many areas also outside of Indian Philosophy, including Legal Representation, see, e.g., Bartolini et al. (2018), Libal and Steen (2020). The main setting here is the following. Suppose that we are given a natural language text, e.g., a passage of a Mīmāmsā text or a specific law or regulation, that we would like to formalize. Because of the ambiguity inherent in natural language as well as, e.g., certain difficulties of interpretation specific to Sanskrit we are almost guaranteed to obtain not a single formalisation, but a number of different competing ones. Hence we are faced with the task of deciding which of these is the most appropriate. 


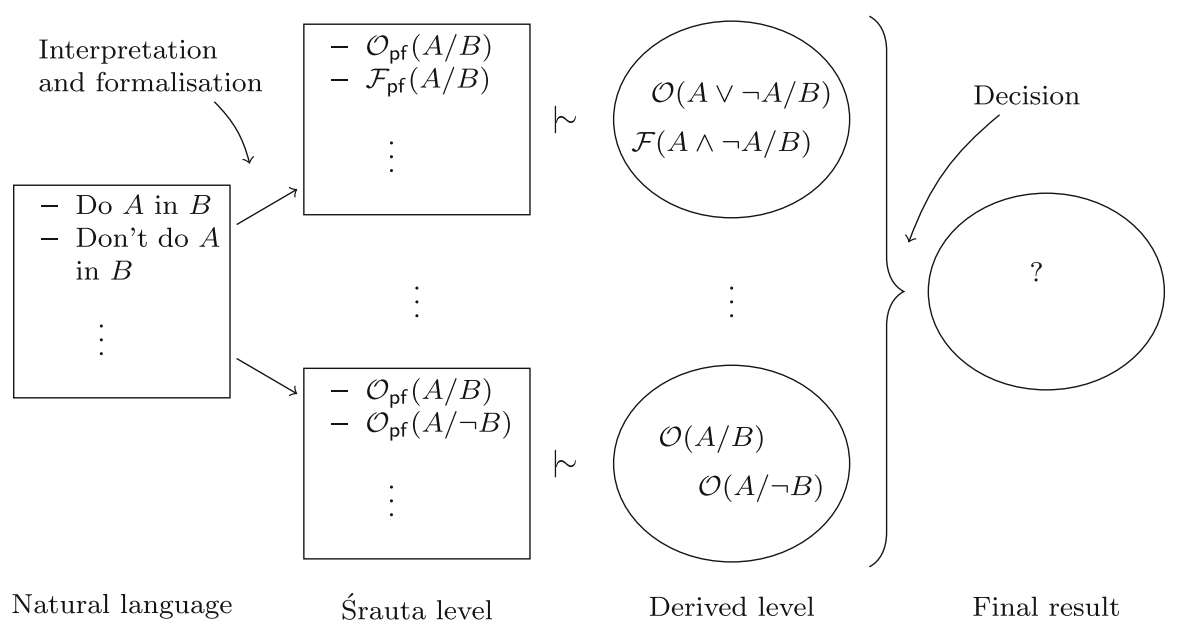

Fig. 17 The procedure for deciding between different interpretations

One way of doing so is to consider the consequences of the different interpretations under an assumed system of background reasoning, in our case the logic MD+, and to compare them with respect to certain criteria. We will see a specific possible criterium used by Mīmāmsā authors below, but in general such criteria would involve a basic sanity check in the form of consistency, or checking whether certain statements, which intuitively should hold, are derivable (compare, e.g., the quality assurance procedures in Libal and Steen 2020). Whenever the principles of the assumed background reasoning match the guiding principles of our system $\mathrm{MD}+$, the decision procedure given above can be used to check the consequences of the different interpretations, and hence aid in comparing them. The general procedure is illustrated in Fig. 17. In view of the fact that in this article our main application is to Mīmāmsā reasoning, in the figure the stage containing the different competing first formalisations is labelled the śrauta level, but it is worth noting again that the procedure itself in principle can be applied to any collection of normative statements, including regulations and laws, as long as the formal language and the assumed system of background reasoning match the ones considered here.

Example 4 Suppose we encounter the following statements:

1. One should refrain from smoking in the presence of a baby.

2. Smoking in a bar incurs a sanction.

3. One may smoke if there is sufficient ventilation.

Further suppose that we have already established that the first of these should be read as an obligation, e.g., in a moral sense, whereas due to the mention of a sanction the second one should be read as a prohibition in the legal sense. The corresponding formalisation would be given by:

1. $\mathcal{O}_{\mathrm{pf}}(\neg$ smoke/baby $)$

2. $\mathcal{F}_{\text {pf }}($ smoke/bar $)$ 
For the third statement, however, it is not clear whether the "may" constitutes an exception to the moral kind of obligation of the first statement or to the legal kind of prohibition of the second. I.e., in the formal language of MD+ we could formalise this statement either as $\mathcal{P}_{\text {pf }}^{\mathcal{O}}$ (smoke/vent) or as $\mathcal{P}_{\text {pf }}^{\mathcal{F}}$ (smoke/vent).

A possible way to decide between these two interpretations is to consider the consequences together with the formalisations of the first two statements. In particular, under the interpretation as $\mathcal{P}_{\text {pf }}^{\mathcal{O}}$ (smoke/vent) and assuming our system of background reasoning, we obtain that $\mathcal{O}(\neg$ smoke/baby $\wedge$ bar $\wedge$ vent $)$ is not derivable, whereas $\mathcal{F}$ (smoke/baby $\wedge$ bar $\wedge$ vent) is, i.e., while it still incurs a legal sanction, from a moral point of view one need not refrain from smoking in the presence of a baby in a well-ventilated bar. In contrast, under the alternative interpretation as $\mathcal{P}_{\text {pf }}^{\mathcal{F}}$ (smoke/vent) we would derive $\mathcal{O}(\neg$ smoke/baby $\wedge$ bar $\wedge$ vent $)$, but would not derive $\mathcal{F}$ (smoke/baby $\wedge$ bar $\wedge$ vent $)$. I.e., in the same situation smoking would not be illegal, but still morally objectionable. Checking these two possible sets of consequences either against our moral intuitions or against information on the legal aspects of smoking would provide us with a method for deciding which of the two possible interpretations of the permission statement would be more plausible.

Similarly to the previous example, we can also use the general procedure of Fig. 17 to decide about assumptions on the level of facts as follows.

Example 5 Suppose that we have a text stipulating that unjustified violence incurs certain sanctions, i.e., is explicitly forbidden. Hence $\mathcal{F}_{\text {pf }}(\mathrm{violence} / \top)$.

Further, suppose that we are interested in the status of suicide. Adding the seemingly plausible fact that suicide is unjustified violence, i.e., suicide $\rightarrow$ violence to the factual assumptions would, in absence of any other prima-facie deontic statements, yield derivability of the formula $\mathcal{F}$ (suicide/T). Thus, if we find evidence that (attempted) suicide is not forbidden, possibly because it does not incur any sanctions, we should conclude that the authors do not consider suicide to be a form of unjustified violence, and hence remove the corresponding factual assumption.

Note that in a certain sense the factual assumptions hence could also be used in a coarse (and perhaps oversimplified) representation of constitutive norms, see, e.g., Boella and van der Torre (2004).

\subsection{The evaluation criterium of vikalpa}

Coming back to Mīmāṃsā reasoning, it is interesting to note that the outlined procedure seems to be the method employed, in an informal manner, by various Mīmāṃāa authors to decide between different interpretations of deontic statements found in the Vedas. One particular decision criterium employed in this context is that of minimising instances of the so-called vikalpa principle. This principle was already stated explicitly in the founding text of the Mīmāmsāa school, the Pūrva Mìmāmsā Sūtra of Jaimini, with the following English translation (and reformulation).

If a prescription enjoins $X$ and a prohibition forbids one to perform the same act $X$ under the same conditions, and no other interpretation is possible, the act $X$ should be considered optional. 
The problem with such optional acts lies in the fact that for the Mīmāmsāa authors if the act is optional, the deontic statement prescribing or prohibiting it would be superfluous in the sense of not being applicable. However, from their point of view the Vedas would not give superfluous information. Hence they see vikalpa as a very last resort, and strive to develop an interpretation of the Vedas which makes use of this device as little as possible.

In our formal framework the discussion around the vikalpa principle has two aspects. The first one is that we should be able to derive it in our system. Abstracting from the particular action $X$ in the quote above, and concentrating on obligations only, this means that, for a set $\mathfrak{L}=\left\{\mathcal{O}_{\mathrm{pf}}(a / b), \mathcal{O}_{\mathrm{pf}}(c / d)\right\}$ and facts $\mathfrak{F}=\{a, c \Rightarrow\}$ establishing that $a$ and $c$ are not jointly possible, neither of the formulae $\mathcal{O}(a / b \wedge d)$ and $\mathcal{O}(c / b \wedge$ $d$ ) should be derivable, because it should be considered optional whether $a$ or $c$ is performed. However, while it is optional which of the two is performed, it is still obligatory to perform one of them, hence the formula $\mathcal{O}(a \vee c / b \wedge d)$ should be derivable.

Remark 7 It is worth noting that more than two millennia after its formulation by Jaimini this principle was also formulated in modern deontic logic and in nonmonotonic reasoning: In the former, it is known, e.g., under the name of disjunctive response, where from the two conflicting assumptions $\mathcal{O}(a / c)$ and $\mathcal{O}(b / c)$ we are able to derive at least the obligation of the disjunction $\mathcal{O}(a \vee b / c)$, see Goble (2013); in the area of nonmonotonic reasoning it roughly corresponds to the phenomenon of floating conclusions for skeptical semantics, where in our example neither of the contents $a$ and $b$ of the two conflicting assumptions would be in all the extensions, but the formula $a \vee b$ is, see Makinson and Schlechta (1991).

Generalising the above example to sets of obligations, and adding that all the enjoined acts should be possible by themselves and that the result should not be blocked by any obligation, prohibition, or permission outside the set, we can derive the vikalpa principle in our system:

Theorem 6 Let $X=\left\{\mathcal{O}_{p f}\left(A_{1} / B_{1}\right), \ldots, \mathcal{O}_{p f}\left(A_{n} / B_{n}\right)\right\} \subseteq \mathfrak{L}$ be a set such that

- (F, $\mathfrak{L}) \nvdash_{G_{M D+} \text { cut }} A_{i} \Rightarrow$ for every $i \leq n$.

- (F, L) $\nvdash_{G_{M D+} \text { cut }} \bigvee_{i \leq n} A_{i}, C \Rightarrow$ for every $\mathcal{O}_{\text {pf }}(C / D) \in \mathfrak{L} \backslash X$ with $(\mathfrak{F}, \mathfrak{L}) \vdash_{G_{M D+} \text { cut }} \bigwedge_{i \leq n} B_{i} \Rightarrow D$.

- (F, $\mathfrak{L}) \nvdash_{G_{M D+} \text { cut }} \bigvee_{i \leq n} A_{i} \Rightarrow C$ for every $\mathcal{F}_{p f}(C / D) \in \mathfrak{L} \backslash X$ with $(\mathfrak{F}, \mathfrak{L}) \vdash_{G_{M D+} \text { cut }}$ $\bigwedge_{i \leq n} B_{i} \Rightarrow D$.

- (F, $\mathfrak{L}) \nvdash_{G_{M D+} \text { cut }} \bigvee_{i \leq n} A_{i}, C \Rightarrow$ for every $\mathcal{P}_{p f}^{\mathcal{O}}(C / D) \in \mathfrak{L} \backslash X$ with $(\mathfrak{F}, \mathfrak{L}) \vdash_{G_{M D+} \text { cut }} \bigwedge_{i \leq n} B_{i} \Rightarrow D$.

Then $(\mathfrak{F}, \mathfrak{L}) \vdash_{G_{M D+} \text { cut }} \Rightarrow \mathcal{O}\left(\bigvee_{i \leq n} A_{i} / \bigwedge_{i \leq n} B_{i}\right)$.

Proof We show that we have all the premises to apply the rule $\mathcal{O}_{R}^{\mathcal{O}_{\text {pf }}\left(A_{1} / B_{1}\right)}$. From the propositional rules we obtain $(\mathfrak{F}, \mathfrak{L}) \vdash_{\mathrm{G}_{\mathrm{MD}+} \text { cut }} A_{1} \Rightarrow \bigvee_{i \leq n}^{R} A_{i}$ and $(\mathfrak{F}, \mathfrak{L}) \vdash_{\mathrm{G}_{\mathrm{MD}+} \text { cut }} \bigwedge_{i \leq n} B_{i} \Rightarrow B_{1}$. Moreover, for every $j \leq n$ we obtain $(\mathfrak{F}, \mathfrak{L}) \nvdash_{\mathrm{G}_{\mathrm{MD}+} \text { cut }} A_{j}, \bigvee_{i \leq n} A_{i} \Rightarrow$, since otherwise in particular we would have 
$(\mathfrak{F}, \mathfrak{L}) \vdash_{\mathrm{G}_{\mathrm{MD}+} \text { cut }} A_{j}, A_{j} \Rightarrow$, and hence $(\mathfrak{F}, \mathfrak{L}) \vdash_{\mathrm{G}_{\mathrm{MD}+} \text { cut }} A_{j} \Rightarrow$. Furthermore, by assumption, for every $\mathcal{O}_{\mathrm{pf}}(C / D) \in \mathfrak{L} \backslash X$ we have either $(\mathfrak{F}, \mathfrak{L}) \nvdash_{\mathrm{G}_{\mathrm{MD}+} \text { cut }} \bigwedge_{i \leq n} B_{i} \Rightarrow$ $D$ or $(\mathfrak{F}, \mathfrak{L}) \nvdash_{\mathrm{G}_{\mathrm{MD}+} \text { cut }} C, \bigvee_{i \leq n} A_{i} \Rightarrow$. The analogous statement holds for every primafacie deontic statement of the form $\mathcal{F}_{\mathrm{pf}}(C / D)$ or $\mathcal{P}_{\mathrm{pf}}^{\mathcal{O}}(C / D)$. Now applying the rule $\mathcal{O}_{R}^{\mathcal{O}_{\text {pf }}\left(A_{1} / B_{1}\right)}$ yields $\Rightarrow \mathcal{O}\left(\bigvee_{i \leq n} A_{i} / \bigwedge_{i \leq n} B_{i}\right)$.

It should be noted that for the statement of the theorem it is not relevant whether the $A_{i}$ from the set $X$ are jointly possible or not, only that their disjunction $\bigvee_{i \leq m} A_{i}$ is not blocked by any $C$ from outside that set. In particular, it also applies to the case where the $A_{i}$ are not jointly possible. Thus, our system as described indeed satisfies the disjunctive response resp. vikalpa. The corresponding statement for prohibitions is shown completely analogously, using the rule $\mathcal{F}_{R}^{\mathcal{F}_{\text {pf }}\left(A_{1} / B_{1}\right)}$ instead of $\mathcal{O}_{R}^{\mathcal{O}_{\text {pf }}\left(A_{1} / B_{1}\right)}$ :

Theorem 7 Let $X=\left\{\mathcal{F}_{p f}\left(A_{1} / B_{1}\right), \ldots, \mathcal{F}_{p f}\left(A_{n} / B_{n}\right)\right\} \subseteq \mathfrak{L}$ be a set such that

- (F, $\mathfrak{L}) \nvdash_{G_{M D+} \text { cut }} \Rightarrow A_{i}$ for every $i \leq n$.

- (F, $\mathfrak{L}) \nvdash_{G_{M D+} \text { cut }} C \Rightarrow \bigwedge_{i \leq n} A_{i}$ for every $\mathcal{O}_{p f}(C / D) \in \mathfrak{L} \backslash X$ with $(\mathfrak{F}, \mathfrak{L}) \vdash_{G_{M D+} \text { cut }}$ $\bigwedge_{i<n} B_{i} \Rightarrow D$.

- (F, $\mathfrak{L}) \nvdash_{G_{M D+} \text { cut }} \Rightarrow \bigwedge_{i \leq n} A_{i}, C$ for every $\mathcal{F}_{p f}(C / D) \in \mathfrak{L} \backslash X$ with $(\mathfrak{F}, \mathfrak{L}) \vdash_{G_{M D+} \text { cut }}$ $\bigwedge_{i \leq n} B_{i} \Rightarrow D$.

$-(\mathfrak{F}, \mathfrak{L}) \nvdash_{G_{M D+} \text { cut }} C \Rightarrow \bigwedge_{i \leq n} A_{i}$ for every $\mathcal{P}_{p f}^{\mathcal{F}}(C / D) \in \mathfrak{L} \backslash X$ with $(\mathfrak{F}, \mathfrak{L}) \vdash_{G_{M D+} \text { cut }}$ $\bigwedge_{i \leq n} B_{i} \Rightarrow D$.

Then $(\mathfrak{F}, \mathfrak{L}) \vdash_{G_{M D+} \text { cut }} \Rightarrow \mathcal{O}\left(\bigwedge_{i \leq n} A_{i} / \bigwedge_{i \leq n} B_{i}\right)$.

The previous theorems show that in our system we can use the fundamental principle of vikalpa to obtain derived deontic statements from conflicting prima-facie statements. But how can we evaluate different interpretations with respect to minimising the number of applications of this principle? At this point it is important to note that what should be minimised is not the number of applications of the vikalpa principle to different derived formulae, but to prima-facie deontic statements, since only superfluousness of the latter is problematic. The general idea then is that a prima-facie deontic statement $\mathcal{O}_{\mathrm{pf}}(a / b)$ or $\mathcal{F}_{\mathrm{pf}}(a / b)$ is involved in an application of the vikalpa principle with another prima-facie deontic statement in a context given by $(\mathfrak{L}, \mathfrak{F})$ exactly when the corresponding formula $\mathcal{O}(a / b)$ or $\mathcal{F}(a / b)$ is not derivable from $(\mathfrak{L}, \mathfrak{F})$. Hence given such a context we can use the decision procedure of Algorithm 1 to identify exactly those prima-facie deontic statements involved in applications of the problematic principle. Apart from providing us with the number of possibly problematic prima-facie formulae, this method has the additional benefit that it explicitly gives these problematic formulae, hence yielding clues as to which parts of the interpretation could be changed in order to avoid applications of the vikalpa principle. Note that this amounts to a form of inconsistency checking using the formalisation of a text similar to that used in Libal and Norotná (2020) for finding and correcting inconsistencies in legal texts This general idea for identifying applications of the vikalpa principle is made formally precise in the following two propositions.

Proposition 4 Let $\mathcal{O}_{p f}(A / B) \in \mathfrak{L}$. Then $(\mathfrak{F}, \mathfrak{L}) \nvdash_{G_{M D+}}$ cut $\Rightarrow \mathcal{O}(A / B)$ holds if and only if at least one of the following holds: 
- There is a $\mathcal{O}_{p f}(C / D) \in \mathfrak{L}$ with $(\mathfrak{F}, \mathfrak{L}) \vdash_{G_{M D+} \text { cut }} A, C \Rightarrow$ and $(\mathfrak{F}, \mathfrak{L}) \vdash_{G_{M D+} \text { cut }} \Rightarrow$ $B \leftrightarrow D$; or

- There is a $\mathcal{P}_{p f}^{\mathcal{O}}(C / D) \in \mathfrak{L}$ with $(\mathfrak{F}, \mathfrak{L}) \vdash_{G_{M D+} \text { cut }} A, C \Rightarrow$ and $(\mathfrak{F}, \mathfrak{L}) \vdash_{G_{M D+} \text { cut }} \Rightarrow$ $B \leftrightarrow D$; or

- There is a $\mathcal{F}_{p f}(C / D) \in \mathfrak{L}$ with $(\mathfrak{F}, \mathfrak{L}) \vdash_{G_{M D+} \text { cut }} A \Rightarrow C$ and $(\mathfrak{F}, \mathfrak{L}) \vdash_{G_{M D+} \text { cut }} \Rightarrow$ $B \leftrightarrow D$.

Proof Using cut elimination and a close inspection of the rules, the only way in which the sequent $\Rightarrow \mathcal{O}(A / B)$ could be derived is via an instance of the assumption rule $\mathcal{O}_{R}^{\mathcal{O}_{\mathrm{pf}}(E / F)}$ for some $\mathcal{O}_{\mathrm{pf}}(E / B) \in \mathfrak{L}$. From this the "if" part follows directly, since in case one of the three conditions hold, not all of the underivability statements in the not-excepted block hold. For the "only if" direction, suppose that $(\mathfrak{F}, \mathfrak{L}) \nvdash_{\mathrm{G}_{\mathrm{MD}+} \mathrm{cut}} \Rightarrow$ $\mathcal{O}(A / B)$ holds. Then in particular, the sequent $\Rightarrow \mathcal{O}(A / B)$ is not derivable via the specific rule $\mathcal{O}_{R}^{\mathcal{O}_{\text {pf }}(A / B)}$. Since the premisses of this rule in the standard block are

$$
B \Rightarrow B \quad \text { and } \quad A \Rightarrow A
$$

which are initial sequents, some of the premisses in the not-excepted block or in the no-active-conflict block must not hold. However, since the formula $\mathcal{O}_{\mathrm{pf}}(A / B)$ is in $\mathfrak{L}$ and overrules any conflicting formula from $\mathfrak{L}$ (since both $B \Rightarrow B$ and $B \Rightarrow Y$ are derivable for any conflicting obligation $\mathcal{O}_{\mathrm{pf}}(X / Y)$, prohibition $\mathcal{F}(X / Y)$, or permission $\mathcal{P}^{\mathcal{O}}(X / Y)$ for which $B \Rightarrow Y$ is derivable), all of the premisses in the no-active-conflict block do hold. Hence some of the premisses in the not-excepted block hold, which means that there is a formula $\mathcal{O}_{\mathrm{pf}}(X / Y), \mathcal{F}_{\mathrm{pf}}(X / Y)$ or $\mathcal{P}_{\mathrm{pf}}^{\mathcal{O}}(X / Y)$ from $\mathfrak{L}$ satisfying the conditions given in the statement of the proposition.

The analogous proposition for prohibitions is proved in the same way:

Proposition 5 Let $\mathcal{F}_{p f}(A / B) \in \mathfrak{L}$. Then $(\mathfrak{F}, \mathfrak{L}) \nvdash_{G_{M D+} \text { cut }} \Rightarrow \mathcal{F}(A / B)$ holds if and only if at least one of the following holds:

- There is a $\mathcal{F}_{p f}(C / D) \in \mathfrak{L}$ with $(\mathfrak{F}, \mathfrak{L}) \vdash_{G_{M D+} \text { cut }} \Rightarrow A, C$ and $(\mathfrak{F}, \mathfrak{L}) \vdash_{G_{M D+} \text { cut }} \Rightarrow$ $B \leftrightarrow D$; or

- There is a $\mathcal{P}_{p f}^{\mathcal{F}}(C / D) \in \mathfrak{L}$ with $(\mathfrak{F}, \mathfrak{L}) \vdash_{G_{M D+} c u t} C \Rightarrow A$ and $(\mathfrak{F}, \mathfrak{L}) \vdash_{G_{M D+} \text { cut }} \Rightarrow$ $B \leftrightarrow D$; or

- There is a $\mathcal{O}_{p f}(C / D) \in \mathfrak{L}$ with $(\mathfrak{F}, \mathfrak{L}) \vdash_{G_{M D+} c u t} C \Rightarrow A$ and $(\mathfrak{F}, \mathfrak{L}) \vdash_{G_{M D+} \text { cut }} \Rightarrow$ $B \leftrightarrow D$.

Using these propositions we can now formally evaluate an interpretation given by a list $\mathfrak{L}$ of prima-facie deontic statements and a set $\mathfrak{F}$ of propositional facts with respect to the criterion of minimising the number of instances of vikalpa among the primafacie deontic statements as follows: For every prima-facie statement $\mathcal{O}_{\mathrm{pf}}(A / B) \in$ $\mathfrak{L}$ and for every $\mathcal{F}_{\text {pf }}(C / D) \in \mathfrak{L}$ check whether $(\mathfrak{F}, \mathfrak{L}) \nvdash_{\mathrm{G}_{\mathrm{MD}+} \text { cut }} \Rightarrow \mathcal{O}(A / B)$ and $(\mathfrak{F}$, $\mathfrak{L}) \nvdash_{\mathrm{G}_{\mathrm{MD}+} \mathrm{cut}} \Rightarrow \mathcal{F}(C / D)$ respectively, and return all those formulae for which this holds. 
The implementation available under http://subsell.logic.at/bprover/deonticProver/ version $1.2 /$ includes this check together with the possibility of automatically generating alternative formalisations by systematically reinterpreting the deontic operators, e.g., by rewriting prohibitions as negative obligations.

The following Mīmāṃsā example arises from the consideration that recommendations and obligations are expressed by the same Sanskrit word: vidhi. Therefore in absence of further discriminating elements the classification of a command could be based on the principle of avoiding vikalpa as much as possible, as shown in the following example.

Example 6 Consider the following simplified interpretation of part of the debate about the Satī sacrifice ${ }^{5}$, discussed in depth in Brick (2010):

(i) "When a woman's husband has died, she should perform the Sati sacrifice by ascending the funeral pyre after him." $\mathcal{O}_{\text {pf }}$ (satî/widow).

(ii) "Every rite which is violence itself is forbidden, therefore the Sati sacrifice for widows is forbidden" $\mathcal{F}_{\text {pf }}$ (sat $\overline{1} /$ widow).

None of these injunctions is derivable in the logic, therefore, under this interpretation, the Satī sacrifice should be considered optional. However some Mīmāmsāa authors propose to interpret the injunction (i) as the recommendation $\mathcal{R}_{\text {pf }}$ (satî/widow), conditioned by a general woman's desire of positive karma for her husband and herself. This explanation should be preferred as not giving rise to cases of vikalpa: the sacrifice remains forbidden, but a woman can chose to perform it for obtaining a desired result; i.e., both $\mathcal{F}$ (satī/widow) and $\mathcal{R}$ (satī/widow) are derivable in the logic.

Another example of how the mechanism can be used for choosing between conflicting interpretations is given by the discussion about permissions: even if they do not appear as operators in MD+, reading a prima-facie permission as an exception to a prohibition $\left(\mathcal{P}_{\text {pf }}^{\mathcal{F}}\right)$ or to an obligation $\left(\mathcal{P}_{\text {pf }}^{\mathcal{O}}\right)$ affects the derivability of the other prescriptions.

Example 7 Consider the following situation which abstracts part of the discussion in Kumārila's Tantravārttika on 1.3.3-4:

(i) "During a particular sacrifice it is forbidden to eat" $\mathcal{F}_{\text {pf }}$ (eat/sacr).

(ii) "In the second part of this sacrifice it is also obligatory (rewarded with good karma) not to eat" $\mathcal{O}_{\mathrm{pf}}(\neg$ eat/sacr_IIpart).

(ii) "In the second part of this sacrifice it is also permitted to eat".

If the permission is considered as an exception to the obligation and formalized as $\mathcal{P}_{\text {pf }}^{\mathcal{O}}$ (eat/sacr_IIpart), then it blocks the derivation of $\mathcal{O}$ ( $\neg$ eat/sacr_IIpart) in the logic. Hence, to ensure that the maximum number of srauta injunctions are derivable in the logic (i.e. the instances of vikalpa are minimized) the permission should be interpreted as an exception to the first prohibition-since, intuitively, sacr_IIpart $\rightarrow$ sacr_and formalized as $\mathcal{P}_{\text {pf }}^{\mathcal{F}}$ (eat/sacr_IIpart). Under this interpretation, eating is forbidden only in the other parts of the sacrifice $\left(\mathcal{F}_{\text {pf }}(\right.$ eat/sacr $)$ is derivable and $\mathcal{F}_{\text {pf }}$ (eat/sacr_IIpart) is not), but it remains obligatory not to eat in the second part ( $\mathcal{O}$ (ᄀeat/sacr_IIpart) is derivable).

\footnotetext{
5 Satī is an old custom where a widow immolates herself on her husband's funeral pyre.
} 


\section{Conclusion}

Focusing on the specificity principle, we have explored connections between the Mīmāmsāa school of Indian philosophy and symbolic deontic logic. We have first extended the basic logic of Mīmāmsāa in Ciabattoni et al. (2015) with new operators for prohibitions and recommendations whose properties have been extracted from the Mīmāmsā texts. The paper's main result is a sequent-based system to reason in this logic using specificity/gunapradhāna; some of its properties have been investigated and its potential use as a tool for Mīmāmsā philosophy as well as to aid formalisation tasks, e.g., in Legal Representation, have been explored.

Future research directions include the extension of the system to deal with further Mīmāmsā rules (nyāyas) to avoid contradictions; we are planning to work on those just discovered in Kumārila's Tantravārttika on 3.3.14 (balābala-adhikarana) which comprise a prioritisation of rules based on an existing hierarchy of sources (e.g. a Vedic prescription defeats a contradictory prescription in the "traditional texts based on the Vedas'), and on the criteria of invalidating as few injunctions as possible.

From the technical side, we plan to investigate the system's semantics, to abstract and generalize it to work for base deontic logics other than MD+ and to further explore its use in other fields, in particular Legal Representation and Reasoning, in detail.

Apart from the technical content, this paper illustrates some of the vast potential for cross-fertilisation between Mīmāṃsā and deontic logic. This enterprise is the subject of ongoing work in collaboration with Sanskritists and experts of Indian philosophy.

Funding Open access funding provided by TU Wien (TUW).

Open Access This article is licensed under a Creative Commons Attribution 4.0 International License, which permits use, sharing, adaptation, distribution and reproduction in any medium or format, as long as you give appropriate credit to the original author(s) and the source, provide a link to the Creative Commons licence, and indicate if changes were made. The images or other third party material in this article are included in the article's Creative Commons licence, unless indicated otherwise in a credit line to the material. If material is not included in the article's Creative Commons licence and your intended use is not permitted by statutory regulation or exceeds the permitted use, you will need to obtain permission directly from the copyright holder. To view a copy of this licence, visit http://creativecommons.org/licenses/by/4.0/.

\section{A Appendix}

Theorem 8 (Cut elimination) If (F, $\mathfrak{L}) \vdash_{G_{M D+} \text { cut }} \Gamma \Rightarrow \Delta$, then $(\mathfrak{F}, \mathfrak{L}) \vdash_{G_{M D+}} \Gamma \Rightarrow \Delta$.

Proof We show how to eliminate topmost applications of the multicut rule

$$
\frac{\Gamma \Rightarrow \Delta, A^{n} A^{m}, \Sigma \Rightarrow \Pi}{\Gamma, \Sigma \Rightarrow \Delta, \Pi} \text { mcut }
$$

from a proto-derivation, preserving validity (here $A^{n}$ is the multiset containing $n$ copies of $A$ ). Since cut is a case of mcut and mcut is derivable using $\operatorname{Con}_{L}, \operatorname{Con}_{R}$ and cut, this suffices. The proof is by double induction on the complexity of the cut formula $A$ and the sum of the depths of the derivations of the two premises of the application 
of mcut (see Troelstra and Schwichtenberg (2000), Sect. 4.1.9 for the classical case without underivability statements).

If the complexity of the cut formula is 0 , then it is a propositional variable, and hence not principal in a modal or propositional rule or a rule from ga $\mathfrak{L}$. Thus, as usual, we permute mcut into the premises of the last applied rules using the inner induction on the depths of the derivations, until it is absorbed by an application of weakening, or reaches the leaves of the proto-derivation. In this case the premises of the multicut are initial sequents or elements of $\mathfrak{F}$. If at least one of these is an initial sequent, the multicut is eliminated as usual, if both sequents are elements of $\mathfrak{F}$ we use that $\mathfrak{F}$ is closed under contraction and cuts and replace the multicut with the corresponding element of $\mathfrak{F}$.

So assume that the complexity of the cut formula is $n+1$. Again, using the inner induction on the depth of the proto-derivation we permute the multicut into the premise(s) of the last applied rules, until it is in an initial sequent or it is principal in the last rules of the derivations of both premises of the multicut. In case the cut formula is propositional we use the standard transformation, see Troelstra and Schwichtenberg (2000).

The only interesting case is where the cut formula is a deontic formula and neither of the two premisses of the multicut is an initial sequent. If the last applied rules both are among $\mathrm{P}_{\mathcal{O}}, \mathrm{P}_{\mathcal{F}}, \mathrm{P}_{\mathcal{R}}, \mathrm{D}_{\mathcal{O}}, \mathrm{D}_{\mathcal{F}}, \mathrm{D}_{\mathcal{O} \mathcal{F}}, \operatorname{Mon}_{\mathcal{O}}, \operatorname{Mon}_{\mathcal{F}}$, Mon $_{\mathcal{R}}$, then the transformation is essentially as for the system $G_{M D}$, see Lellmann and Pattinson (2013) for the general transformations. E.g., if the last applied rules were Mon $_{\mathcal{O}}$ and $\mathrm{D}_{\mathcal{O}}$, the multicut has the following form:

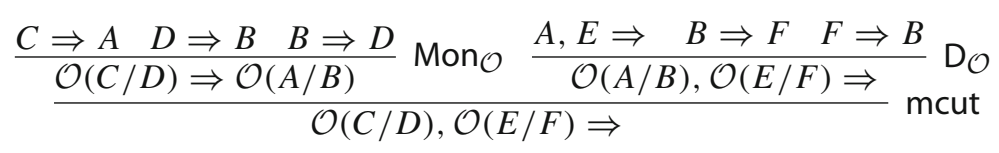

Using the induction hypothesis on the complexity of the cut formula we obtain valid proto-derivations of the conclusions of

$$
\frac{C \Rightarrow A \quad A, E \Rightarrow}{C, E \Rightarrow} \text { mcut } \frac{D \Rightarrow B \quad B \Rightarrow F}{D \Rightarrow F} \text { mcut } \frac{F \Rightarrow B \quad B \Rightarrow D}{F \Rightarrow D} \text { mcut }
$$

Now an application of the rule $\mathrm{D}_{\mathcal{O}}$ yields the sequent $\Gamma, \mathcal{O}(C / D), \Sigma, \mathcal{O}(E / F) \Rightarrow$ $\Delta, \Pi$. In case both principal formulae of the application of $\mathrm{D}_{\mathcal{O}}$ are cut formulae, we proceed similarly, only using the rule $\mathrm{P}_{\mathcal{O}}$ in the last step. The other cases of the modal rules are similar.

In the most interesting cases at least one of the premises of the cut was derived using a rule from ga $\mathrm{g}_{\mathfrak{L}}$. For each operator op $\in\{\mathcal{O}, \mathcal{F}, \mathcal{R}\}$ there are three major groups of cases: (i) $\mathrm{op}_{R}^{\mathrm{op}^{\prime}(C / D)}$ or $\mathrm{op}_{L}^{\mathrm{op}^{\prime}(C / D)}$ versus a rule not from ga $\mathfrak{L}$ where the multicut has non-empty conclusion; (ii) $\mathrm{op}_{R}^{\mathrm{op}^{\prime}(C / D)}$ or $\mathrm{op}_{L}^{\mathrm{op}^{\prime}(C / D)}$ versus a rule not from ga $\mathrm{L}_{\mathfrak{L}}$ where the multicut has an empty conclusion; or (iii) $\mathrm{op}_{R}^{\mathrm{op}^{\prime}(C / D)}$ versus $\mathrm{op}_{L}^{\mathrm{op}^{\prime}(G / H)}$. We consider all the different cases for op $=\mathcal{O}$. The cases for the operators $\mathcal{F}$ and $\mathcal{R}$ are analogous, and much simpler in the case of $\mathcal{R}$. 
Case (i) The prime example of this case is the case where the two last applied rules

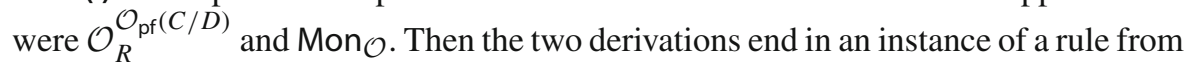

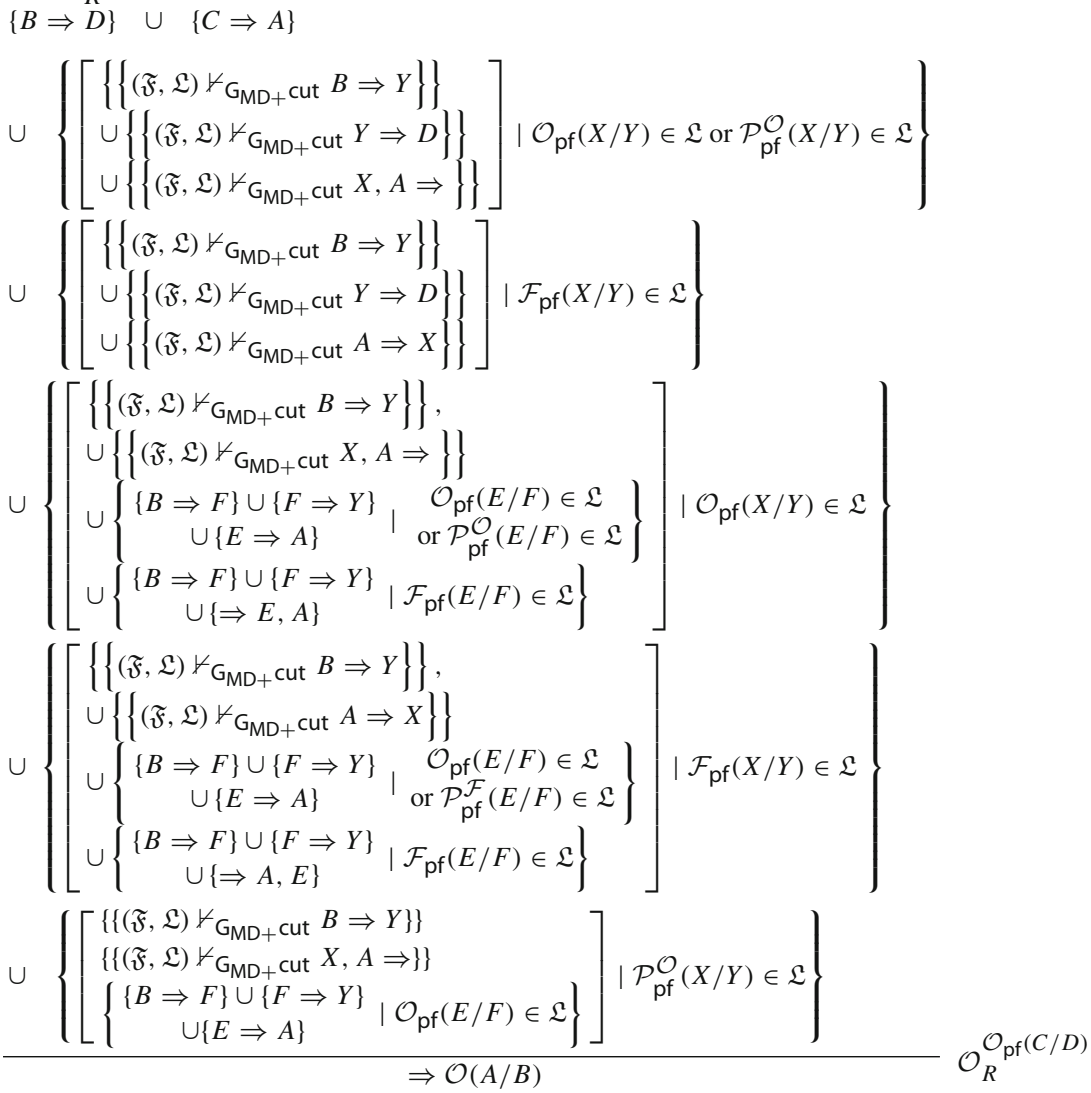

and

$$
\frac{A \Rightarrow G \quad B \Rightarrow H \quad H \Rightarrow B}{\mathcal{O}(A / B) \Rightarrow \mathcal{O}(G / H)} \operatorname{Mon}_{\mathcal{O}}
$$

respectively. By induction hypothesis on the complexity of the cut formula we obtain valid proto-derivations of $H \Rightarrow D$ and $C \Rightarrow G$, as well as the sequents $H \Rightarrow$ $F$ and $F \Rightarrow Y$ and $E \Rightarrow G$ whenever the corresponding sequents occur in the application of $\mathcal{O}_{R}^{\mathcal{O}_{\text {pf }}(C / D)}$. Further, for every underivability statement $(\mathfrak{F}, \mathfrak{L}) \nvdash_{\mathrm{G}_{\mathrm{MD}+} \text { cut }}$ $B \Rightarrow Y$ together with derivability of $B \Rightarrow H$ we obtain the underivability statement $(\mathfrak{F}, \mathfrak{L}) \nvdash_{\mathrm{G}_{\mathrm{MD}+} \text { cut }} H \Rightarrow Y$ by contradiction: assuming there is a valid proto-derivation of $H \Rightarrow Y$ in $\mathrm{G}_{\mathrm{MDga}}$ ${ }_{\mathfrak{L}}$ cut from $\mathfrak{F}$ we could apply cut to this and $B \Rightarrow H$ to obtain $\mathfrak{F} \vdash \vdash_{\mathrm{MD}} a_{\mathcal{L}}$ cut $B \Rightarrow Y$, in contradiction to $(\mathfrak{F}, \mathfrak{L}) \nvdash_{\mathrm{G}_{\mathrm{MD}+} \text { cut }} B \Rightarrow Y$. Similarly, for every underivability statement $(\mathfrak{F}, \mathfrak{L}) \nvdash_{\mathrm{G}_{\mathrm{MD}+} \text { cut }} X, A \Rightarrow$ using derivability of $A \Rightarrow G$ we obtain the underivability statement $(\mathfrak{F}, \mathfrak{L}) \nvdash_{\mathrm{G}_{\mathrm{MD}+} \text { cut }} X, G \Rightarrow$; analogously for the underivability statements $(\mathfrak{F}, \mathfrak{L}) \nvdash_{\mathrm{G}_{\mathrm{MD}+} \text { cut }} A \Rightarrow X$ using derivability of $A \Rightarrow G$ we 
get $(\mathfrak{F}, \mathfrak{L}) \nvdash_{\mathrm{G}_{\mathrm{MD}+} \text { cut }} G \Rightarrow X$. Hence we can apply the rule $\mathcal{O}_{R}^{\mathcal{O}_{\mathrm{pf}}(C / D)}$ to obtain a protoderivation of $\Rightarrow \mathcal{O}(G / H)$. By the reasoning above, all the underivability statements hold, hence the proto-derivation is valid.

The cases where the two last applied rules were $\mathcal{O}_{R}^{\mathcal{O}_{\text {pf }}(C / D)}$ and $\mathrm{D}_{\mathcal{O}}$ with only one of the principal formulae a cut formula or $\operatorname{Mon}_{\mathcal{O}}$ and $\mathcal{O}_{L}^{\mathcal{O}_{\text {pf }}(C / D)}$ are similar, in each case finishing with an application of $\mathcal{O}_{L}^{\mathcal{O}_{\text {pf }}(C / D)}$.

Similarly, in the case where the last applied rules were $\mathcal{O}_{R}^{\mathcal{O}_{\text {pf }}(C / D)}$ and $\mathrm{D}_{\mathcal{O F}}$

we reason as above, but finishing with an application of $\mathcal{F}_{L}^{\mathcal{O}(C / D)}$.

The case where the last applied rules were $\operatorname{Mon}_{\mathcal{O}}$ and $\mathcal{O}_{L}^{\mathcal{F}_{\text {pf }}(C / D)}$ is also similar, finishing with an application of $\mathcal{O}_{L}^{\mathcal{F}_{\text {pf }}(C / D)}$.

Case (ii) The prime example for this case is when the last rules were $\mathcal{O}_{R}^{\mathcal{O}_{\text {pf }}(C / D)}$ and $\mathrm{P}_{\mathcal{O}}$. We claim that this case actually cannot occur. For otherwise the derivations end in an instance of (1) and

$$
\frac{A \Rightarrow}{\mathcal{O}(A / B) \Rightarrow} \mathrm{P}_{\mathcal{O}}
$$

However, then for $X:=C$ and $Y:=D$ we have valid proto-derivations for all three of $B \Rightarrow Y$ and $Y \Rightarrow D$ and $X, A \Rightarrow$. The first one is the first premise of the application of $\mathcal{O}_{R}^{\mathcal{O}_{\text {pf }}(C / D)}$, the second one is easily derivable since $Y=D$, and the last one follows from the premise of $\mathrm{P}_{\mathcal{O}}$ using $\mathrm{W}_{L}$. But then the proto-derivation of $\Rightarrow \mathcal{O}(A / B)$ cannot have been valid since for some of the underivability statements in the not-excepted block of the premisses of the rule $\mathcal{O}_{R}^{\mathcal{O}_{\text {pf }}(C / D)}$ there is a valid proto-derivation.

The case where the last rules were $\mathcal{O}_{R}^{\mathcal{O}_{\text {pf }}(C / D)}$ and $\mathrm{D}_{\mathcal{O}}$ with both principal formulae of the latter cut formulae is analogous to the previous case.

Case (iii) Assume that both last applied rules are from ga $\mathfrak{L}_{\mathfrak{L}}$. The prime example of this is where the last rules were $\mathcal{O}_{R}^{\mathcal{O}_{\mathrm{pf}}(C / D)}$ and $\mathcal{O}_{L}^{\mathcal{O}_{\mathrm{pf}}(G / H)}$. Again, we claim that this cannot happen. For suppose it did, then the derivations would end in (1) and 


$$
\begin{aligned}
& \{B \Rightarrow H\} \quad \cup \quad\{G, A \Rightarrow\} \\
& \cup\left\{\left[\begin{array}{l}
\left\{\left\{(\mathfrak{F}, \mathfrak{L}) \nvdash_{\mathrm{G}_{\mathrm{MD}+} \text { cut }} B \Rightarrow Y\right\}\right\} \\
\cup\left\{\left\{(\mathfrak{F}, \mathfrak{L}) \nvdash_{\mathrm{G}_{\mathrm{MD}+} \text { cut }} Y \Rightarrow H\right\}\right\} \\
\cup\left\{\left\{(\mathfrak{F}, \mathfrak{L}) \nvdash_{\mathrm{G}_{\mathrm{MD}+} \text { cut }} X \Rightarrow A\right\}\right\}
\end{array}\right] \mid \mathcal{O}_{\mathrm{pf}}(X / Y) \in \mathfrak{L} \text { or } \mathcal{P}_{\mathrm{pf}}^{\mathcal{O}}(X / Y) \in \mathfrak{L}\right\} \\
& \cup\left\{\left[\begin{array}{l}
\left\{\left\{(\mathfrak{F}, \mathfrak{L}) \nvdash_{\mathrm{G}_{\mathrm{MD}+\mathrm{cut}}} B \Rightarrow Y\right\}\right\} \\
\cup\left\{\left\{(\mathfrak{F}, \mathfrak{L}) \nvdash_{\mathrm{G}_{\mathrm{MD}+\mathrm{cut}}} Y \Rightarrow H\right\}\right\} \\
\cup\left\{\left\{(\mathfrak{F}, \mathfrak{L}) \nvdash_{\left.\left.\mathrm{G}_{\mathrm{MD}+\mathrm{cut}} \Rightarrow A, X\right\}\right\}}\right.\right.
\end{array}\right] \mid \mathcal{F}_{\mathrm{pf}}(X / Y) \in \mathfrak{L}\right\}
\end{aligned}
$$

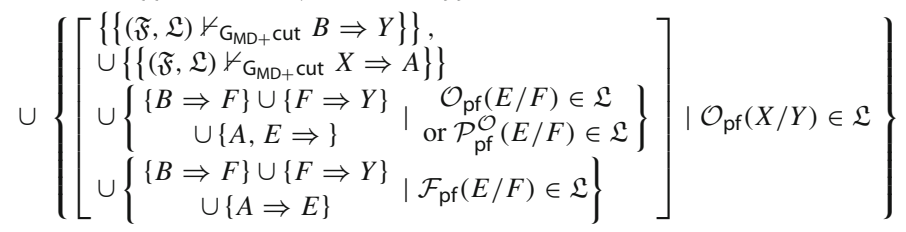

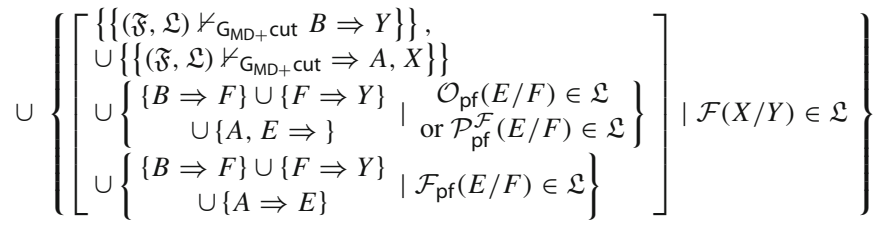

$$
\begin{aligned}
& \frac{\left\{\left[\begin{array}{l}
\left\{\left\{(\mathfrak{F}, \mathfrak{L}) \nvdash_{\mathrm{G}_{\mathrm{MD}+} \text { cut }} B \Rightarrow Y\right\}\right\} \\
\left\{\left\{(\mathfrak{F}, \mathfrak{L}) \nvdash \mathrm{G}_{\mathrm{MD}+\mathrm{cut}} X \Rightarrow A\right\}\right\} \\
\left.\left\{\begin{array}{c}
\{B \Rightarrow F\} \cup\{F \Rightarrow Y\} \\
\cup\{E, A \Rightarrow\}
\end{array}\right] \mid \mathcal{O}_{\mathrm{pf}}(E / F) \in \mathfrak{L}\right\}
\end{array}\right] \mid \mathcal{P}_{\mathrm{pf}}^{\mathcal{O}}(X / Y) \in \mathfrak{L}\right\}}{\mathcal{O}(A / B) \Rightarrow} \mathcal{O}_{L}^{\mathcal{O}_{\mathrm{pf}}(G / H)}
\end{aligned}
$$

But then in particular the no-active conflict block of the application of the rule $\mathcal{O}_{R}^{\mathcal{O}_{\mathrm{pf}}(C / D)}$ has either (a) one of the premises (F, $\left.\mathfrak{L}\right) \nvdash_{\mathrm{G}_{\mathrm{MD}+} \text { cut }} B \Rightarrow H$ and $(\mathfrak{F}, \mathfrak{L}) \nvdash_{\mathrm{G}_{\mathrm{MD}+} \text { cut }} G, A \Rightarrow$; or (b) all of the three premises

$$
B \Rightarrow F \quad F \Rightarrow H \quad E \Rightarrow A
$$

for some $\mathcal{O}_{\mathrm{pf}}(E / F)$ or $\mathcal{P}_{\mathrm{pf}}^{\mathcal{O}}(E / F)$ from $\mathfrak{L}$; or (c) all of the three premises

$$
B \Rightarrow F \quad F \Rightarrow H \quad \Rightarrow A, E
$$

for some $\mathcal{F}_{\mathrm{pf}}(E / F)$ from $\mathfrak{L}$. However, the first case of (a) gives a contradiction with the premise $B \Rightarrow H$ of the application of $\mathcal{O}_{L}^{\mathcal{O}_{\text {pf }}(G / H)}$ using validity of the proto-derivation. The second case gives a contradiction with the premise $G, A \Rightarrow$ of $\mathcal{O}_{L}^{\mathcal{O}_{\text {pf }}(G / H)}$, again using validity of the proto-derivation. Case (b) gives a contradiction because the notexcepted block of the application of $\mathcal{O}_{L}^{\mathcal{O}_{\text {pf }}(G / H)}$ contains one of the premises

$$
(\mathfrak{F}, \mathfrak{L}) \nvdash_{\mathrm{G}_{\mathrm{MD}+} \text { cut }} B \Rightarrow F \quad(\mathfrak{F}, \mathfrak{L}) \nvdash_{\mathrm{G}_{\mathrm{MD}+} \text { cut }} F \Rightarrow H \quad(\mathfrak{F}, \mathfrak{L}) \nvdash_{\mathrm{G}_{\mathrm{MD}+} \text { cut }} E \Rightarrow A
$$

and the proto-derivation is valid. Case (c) is similar, but with (F, $\mathfrak{L}) \nvdash_{\mathrm{G}_{\mathrm{MD}+} \text { cut }} \Rightarrow A, E$ instead of the last underivability statement. Hence this case also cannot occur.

The case of $\mathcal{O}_{R}^{\mathcal{O}_{\text {pf }}(C / D)}$ versus $\mathcal{O}_{L}^{\mathcal{F}_{\text {pf }}(G / H)}$ is analogous. 


\section{References}

Abraham M, Gabbay DM, Schild U (2011) Obligations and prohibitions in Talmudic deontic logic. Artif Intell Law 19(2-3):117-148

Bartolini C, Lenzini G, Santos C (2018) An agile approach to validate a formal representation of the GDPR. In: Kazuhiro K, Maki S, Koji M, Ken S (eds) New frontiers in artificial intelligence. JSAI-isAI 2018, volume 11717 of LNCS. Springer, pp 160-176

Bathia KL (2010) Legal language and legal writing. Universal Law Publishing Co, New Delhi

Batsakis S, Baryannis G, Governatori G, Tachmazidis I, Antoniou G (2018) Legal representation and reasoning in practice: a critical comparison. In: Legal knowledge and information_JURIX 2018. IOS Press, pp 31-40

Boella G, van der Torre LWN (2004) Regulative and constitutive norms in normative multiagent systems. In: Didier D, Christopher AW, Mary-Anne W (eds) Principles of knowledge representation and reasoning: proceedings of the ninth international conference (KR2004), June 2-5, 2004. AAAI Press, Whistler, Canada, pp 255-266

Bonatti PA, Olivetti N (2002) Sequent calculi for propositional nonmonotonic logics. ACM Trans Comput $\log 3(2): 226-278$

Brick D (2010) The Dharmaśāstric debate on widow-burning. J Am Orient Soc 130(2):203-223

Calegari R, Contissa G, Lagioia F, Omicini A, Sartor G (2019) Defeasible systems in legal reasoning: a comparative assessment. In: Legal knowledge and information systems. IOS Press, pp 169-174

Chandra AK, Kozen DC, Stockmeyer LJ (1981) Alternation. J Assoc Comput Mach 28(1):114-133

Chellas BF (1980) Modal logic. Cambridge University Press, Cambridge

Ciabattoni A, Freschi E, Genco FA, Lellmann B (2015) Mīmāmsā deontic logic: proof theory and applications. In: TABLEAUX 2015, volume 9323 of LNCS. Springer, pp 323-338

Ciabattoni A, Freschi E, Genco FA, Lellmann B (2017) Understanding prescriptive texts: rules and logic as elaborated by the Mīmāmsā school. Online J World Philos 2:47-66

Ciabattoni A, Gulisano F, Lellmann B (2018) Resolving conflicting obligations in Mīmāmsā: a sequentbased approach. In: Jan B, Cleo C, Shyam N, Gabriella P (eds) DEON 2018 proceedings. College Publications, pp 91-109

Delgrande JP, Schaub TH (1997) Compiling specificity into approaches to nonmonotonic reasoning. Artif Intell 90(1):301-348

Freschi E (2012) Duty, language and exegesis in Prābhākara Mīmāmsā: including an edition and translation of Rāmānujācārya's Tantrarahasya, Śāstraprameyapariccheda. Number 17 in Jerusalem studies in religion and culture. Brill, Leiden

Freschi E (2017) Mīmāmsā. In: Bilimoria P (ed) History of Indian philosophy. Routledge, London, pp $148-156$

Freschi E, Ollett A, Pascucci M (2019) Duty and sacrifice: a logical analysis of the Mīmāmsā theory of vedic injunctions. Hist Philos Log 40:323-354

Gabbay DM (1985) Theoretical foundations for non-monotonic reasoning in expert systems. In: Logics and models of concurrent systems. Springer, pp 439-457

Goble L (2013) Prima facie norms, normative conflicts, and dilemmas. In: Gabbay D, Horty J, Parent X, van der Meyden R, van der Torre L (eds) Handbook of deontic logic and normative systems. College Publications, pp 241-351. http://www.collegepublications.co.uk/handbooks/?00001

Goble L (2019) Axioms for Hansson's dyadic deontic logics. Filosofiska Notiser 6(1):13-61

Governatori G, Rotolo A (2004) Defeasible logic: agency, intention and obligation. In: Alessio L, Donald N (eds) Deontic logic in computer science, volume 3065 of LNCS. Springer, pp 114-128

Governatori G, Rotolo A (2006) Logic of violations: a Gentzen system for reasoning with contrary-to-duty obligations. Australas J Log 4:193-215

Hage J (2003) Law and defeasibility. Artif Intell Law 11:221-243

Hansson B (1969) An analysis of some deontic logics. Noûs 3(4):373-398

Horty JF (1993) Deontic logic as founded on nonmonotonic logic. Ann Math Artif Intell 9(1-2):69-91

Horty JF (2012) Reasons as defaults. Oxford University Press, Oxford

Huisjes CH (1981) Norms and logic. Thesis. University of Groningen

Jones AJI, Sergot M (1992) Deontic logic in the representation of law: towards a methodology. Artif Intell Law 1:45-64

Katju M (2006) The mīmāmsā principles of interpretation. In: Krishnacharya TP (ed) Pūrvamīmīmāmsā from an interdisciplinary point of view, volume II of history of science, philosophy, and culture in 
Indian civilization: life, thought, and culture in India. Munshiram Manoharlal Publishers Pvt. Ltd., New Delhi, pp 615-625

Lellmann B, Pattinson D (2013) Constructing cut free sequent systems with context restrictions based on classical or intuitionistic logic. In: ICLA 2013, volume 7750 of LNCS. Springer, pp 148-160

Libal T, Norotná T (2020) Towards automating inconsistency checking of legal texts. In: Jusletter IT: IRIS 2020. Editions Weblaw. https://doi.org/10.38023/336778dc-530a-48ac-95da-336a8bd40995

Libal T, Pascucci M (2019) Automated reasoning in normative detachment structures with ideal conditions. In: ICAIL 2019. ACM, pp 63-72

Libal T, Steen A (2020) NAI: towards transparent and usable semi-automated legal analysis. In: Jusletter IT: IRIS 2020. Editions Weblaw, pp 265-272. https://doi.org/10.38023/2eb63e02-f13e-45f5-9a7bd7fe $55 \mathrm{e} 42 \mathrm{c} 6 \mathrm{c}$

Makinson D, Schlechta K (1991) Floating conclusions and zombie paths: two deep difficulties in the 'directly skeptical' approach to inheritance nets. Artif Intell 48:199-209

Makinson D, van der Torre L (2000) Input/output logics. J Philos Log 29(4):383-408

Nute D (1997) Apparent obligation. In: Nute D (ed) Defeasible deontic logic. Springer, Dordrecht, pp 287-315

Nute D (2003) Defeasible logic. In: INAP 2001, volume 2543 of LNCS. Springer, pp 151-169

Prakken H, Sartor G (1999) A system for defeasible argumentation, with defeasible priorities. In: Wooldridge MJ, Veloso MM (eds) Artificial intelligence today: recent trends and developments, volume 1600 of lecture notes in computer science. Springer, pp 365-379

Prakken H, Sergot M (1997) Dyadic deontic logic and contrary-to-duty obligations. In: Nute D (ed) Defeasible deontic logic. Kluwer, Dordrecht, pp 223-262

Royakkers LMM (1998) Extending deontic logic for the formalisation of legal rules, volume 36 of law and philosohpy library. Springer

Straßer C, Antonelli GA (2016) Non-monotonic logic. In: Zalta EN (ed) The Stanford encyclopedia of philosophy (Fall 2016 Edition). Stanford University, pp 1-62. https://plato.stanford.edu/archives/ fall2016/entries/logic-nonmonotonic/

Straßer C, Arieli O (2019) Normative reasoning by sequent-based argumentation. J Log Comput 29(3):381415

Taber J (2004) Is Indian logic nonmonotonic? Philos East West 54:143-170

Troelstra AS, Schwichtenberg H (2000) Basic proof theory, 2nd edn. Cambridge University Press, Cambridge

van der Torre LWN (1994) Violated obligations in a defeasible deontic logic. In: ECAI 94. Wiley, pp 371-375

van Fraassen BC (1972) The logic of conditional obligation. J Philos Log 1(3-4):417-438

von Wright GH (1964) A new system of deontic logic. Dan Yearb Philos 1:173-182

von Wright GH (1965) A correction to a new system of deontic logic. Dan Yearb Philos 2:103-107 